\title{
1 Determining protein structures using genetics
}

2 Jörn M. Schmiedel ${ }^{1}$, Ben Lehner ${ }^{1-3^{*}}$

$3{ }^{1}$ Systems Biology Program, Centre for Genomic Regulation (CRG), The Barcelona Institute of

4 Science and Technology, Dr. Aiguader 88, Barcelona 08003, Spain

$5 \quad 2$ Universitat Pompeu Fabra (UPF), Barcelona 08003, Spain

$6{ }^{3}$ Institució Catalana de Recerca i Estudis Avançats (ICREA), Pg. Lluís Companys 23, 08010

7 Barcelona, Spain

$8 \quad$ *email: ben.lehner@crg.eu

\section{Summary}

11 Determining the three dimensional structures of macromolecules is a major goal of biological

12 research because of the close relationship between structure and function. Structure 13 determination usually relies on physical techniques including x-ray crystallography, NMR 14 spectroscopy and cryo-electron microscopy. Here we present a method that allows the high15 resolution three-dimensional structure of a biological macromolecule to be determined only from 16 measurements of the activity of mutant variants of the molecule. This genetic approach to 17 structure determination relies on the quantification of genetic interactions (epistasis) between 18 mutations and the discrimination of direct from indirect interactions. This provides a new 19 experimental strategy for structure determination, with the potential to reveal functional and in 20 vivo structural conformations at low cost and high throughput. 


\section{Introduction}

23 Mutations within a protein or RNA can have non-independent effects on fitness ${ }^{1-4}$. Indeed, the

24 effects of double mutants have long been used to probe the energetic couplings between positions in a protein to understand determinants of protein folding and stability ${ }^{5,6}$. Early work revealed that at least some strongly interacting positions within a protein are in direct structural contact ${ }^{5-8}$. Deep mutagenesis of proteins ${ }^{9-12}$ and RNAs ${ }^{13-16}$ has further confirmed this conclusion that some - but by no means all - genetic (or epistatic) interactions occur between structurally proximal mutations.

Further support for the idea that non-independence between mutations provides structural information comes from the analysis of amino acid and nucleotide sequence evolution. Here, correlated pairs of amino acids or nucleotides in multiple sequence alignments identify coevolving positions within proteins and RNAs ${ }^{17-19}$. These patterns of co-evolution have been used to identify energetically-coupled positions and 'sectors' within proteins ${ }^{20,21}$. Moreover, when very large numbers of homologous proteins and RNAs are avaiable in sequence databases, the application of global statistical models has proven sufficient to discriminate direct structural contacts from patterns of co-evolution ${ }^{22-24}$, allowing the prediction of macromolecular structures and interactions ${ }^{25-34}$.

Could epistatic interactions quantified from deep mutational scanning experiments be used to determine macromolecular structures? If successful, structure determination by deep mutagenesis would offer a number of advantages over established techniques. First, it requires no specialized equipment or expertise beyond the ability to mutate a molecule, select functional variants, and quantify enrichments by sequencing. Appropriate in vitro and in vivo selection assays already exist for very many molecules of interest and generic assays based on folding, stability, and physical interactions have also been developed ${ }^{9,35-38}$. Second, it could be applied to molecules whose structures are difficult to determine by physical techniques such as intrinsically disordered and membrane proteins. Third, unlike evolutionary coupling analysis

48 there is no requirement for large numbers of homologous sequences and so it could be applied to fast-evolving, recently-evolved and de novo designed proteins and RNAs ${ }^{26,32,39}$. Finally, and

50 perhaps most importantly, it would provide a general strategy to determine the physiologically 51 relevant structures of molecules whilst they are performing particular functions that can be 52 selected for, including in vivo within cells. A cheap and straightforward approach for studying macromolecular structures in vivo would be a very exciting new frontier for cell biology. 
54 Here we show that deep mutational scanning (DMS) of proteins can provide sufficient

55 information to determine their high-resolution three-dimensional structures. Our statistical

56 approach quantifies how often mutations between positions interact epistatically and how such

57 epistatic interaction patterns correlate. These metrics accurately identify individual tertiary

58 structure contacts as well as secondary structure elements within a protein. The same

59 approach also identifies contacts between protein interaction partners. DMS data alone

60 suffices to determine protein structures with accuracies down to $1.9 \AA$ backbone root mean

61 square deviation (RMSD) compared to known reference structures. Moreover, we show that

62 deep learning can further improve prediction performance, allowing the use of much sparser

63 and lower quality DMS datasets for structure determination. This approach therefore provides a

64 new experimental strategy for structure determination that can reveal functional and in vivo

65 structural conformations at low cost and high throughput.

66

67 


\section{Results}

\section{Epistasis is enriched in but not exclusive to structural contacts}

70 To investigate how genetic - or epistatic - interactions between mutations in a protein relate to

71 structure we first used deep mutational scanning data for the immunoglobulin-binding protein $\mathrm{G}$

72 B1 domain (GB1) generated by Olson, et al. ${ }^{11}$. This dataset is the most complete double mutant

73 deep mutagenesis of a protein domain reported to date and was generated by replacing each of

7455 residues of the wild-type domain with all 19 alternative amino acids both individually and as

75 double mutant pairwise combinations, resulting in a library of more than half a million variants

$76\left(55^{*} 19=1,045\right.$ single mutants plus nearly $55^{*} 54 / 2 * 19 * 19=536,085$ double mutants $)$. mRNA

77 display was used to combine an in vitro immunoglobulin $\mathrm{G}$ binding assay with a sequencing

78 readout to determine protein fitness via changes in variant frequencies in the library before and

79 after binding (Extended Data Figure 1, steps 1-4); resulting in a two orders of magnitude

80 measurement range with a median relative error of fitness estimates of $2.8 \%$ (see Figure $2 \mathrm{~A}$,

81 Table 1 and Methods).

82 We first computed which double mutant variants show epistatic fitness effects, i.e. non-

83 independent fitness effects of the constituting single mutant variants (Figure 1B). Non-specific

84 dependencies between mutants might be introduced by non-linearities in the fitness assay,

85 systematic biases in error magnitudes as well as non-specific epistatic behavior, e.g. from

86 thermodynamic stability effects ${ }^{1,9}$. We thus applied a non-parametric null model - the running

87 median of double mutant fitness values given the constituting single mutant fitness values - for

88 the independence of mutations. Equivalently, we calculated 5th and 95th percentile fitness

89 surfaces; and classified double mutants with fitness lower than the $5^{\text {th }}$ percentile as negative

90 epistatic and double mutants with fitness higher than the $95^{\text {th }}$ percentile as positive epistatic. We

91 restricted the evaluation of positive or negative epistasis, however, to specific subsets of the

92 data, where measurement errors do not impede epistasis classification (Extended Data Figure

$932 \mathrm{C}$, see Methods), which results in about $80 \%$ and $55 \%$ of double mutants being suitable for

94 positive or negative epistasis classification, respectively, with a lot of variability across the

95 position matrix (Extended Data Figures 2D-F and Table 1).

96 Consistent with previous observations ${ }^{10-12}$, both positive and negative epistatic double mutants

97 are enriched for proximal variants, for example, more than 2-fold at $8 \AA$ distance (side-chain

98 heavy atom minimal distance, Figure 1C). However, about $75 \%$ of epistatic interactions are 
between positions that are not in direct contact in the tertiary protein structure (as judged by an $8 \AA$ distance cutoff), suggesting that indirect effects often underlie epistatic interactions within a molecule ${ }^{20,21}$. The challenge for structure determination therefore becomes how to infer direct structural contacts from the mixture of direct and indirect effects that must underlie epistasis.

\section{Figure 1}

A

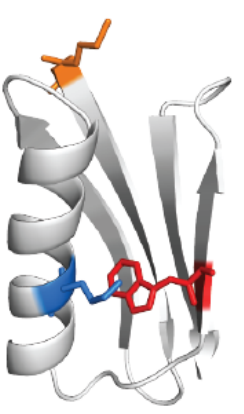

distal: proximal: no epistasis epistasis

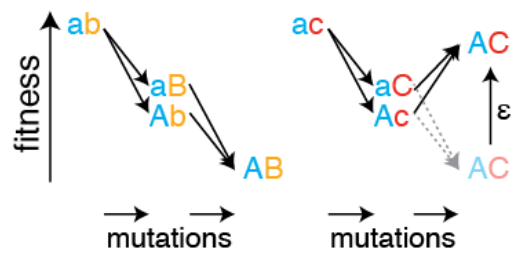

B

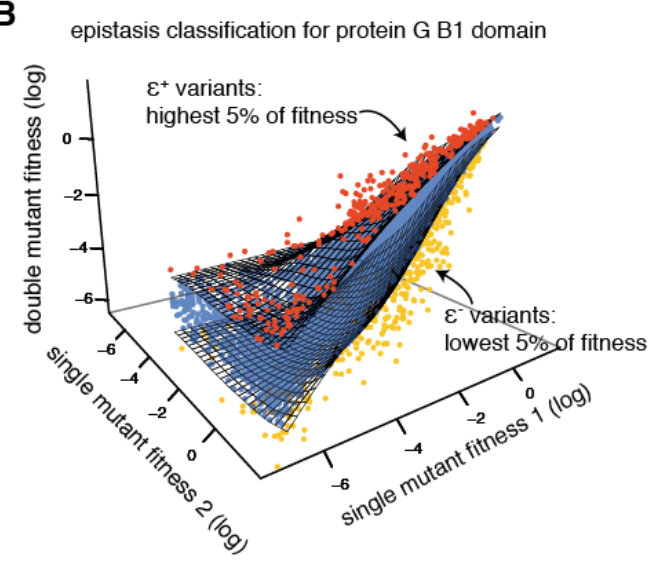

C

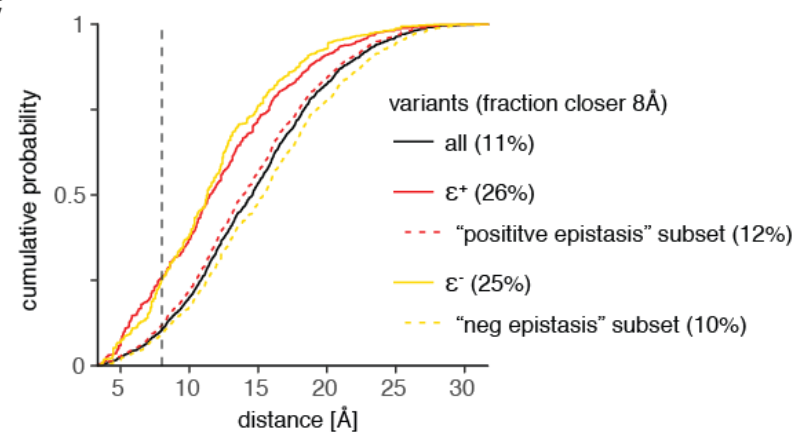

A. Premise: If epistatic interactions relate to structural contacts then quantifying epistatic interactions should suffice to predict a molecule's structure. Structure: protein G B1 domain (PDB entry: 1pga) with residues a, b, and c colored.

B. Classifying epistatic variants based on deviations from expected fitness (based on quantile fitness surface approach). Variants above the 95th or below the 5th percentile of double mutant fitness given their single mutant fitness values were classified as positive (red, $\varepsilon^{+}$) or negative (yellow, $\varepsilon^{-}$) epistatic, respectively. Shown is a random sample of $10^{4}$ variants in GB1 domain ${ }^{11}$. 
C. Distance distribution of epistatic variants separated by more than 5 amino acids in the linear sequence. (side-chain heavy atom distance in reference structure). Positive and negative epistasis subsets refer to the sets of variants applicable for epistasis analysis (see Extended Data Figure 2C). All variants, $n=400647$; positive epistatic variants $\varepsilon^{+}, n$ $=14127$; positive epistasis subset, $\mathrm{n}=315862$; negative epistatic variants $\varepsilon^{-}, \mathrm{n}=9837$; negative epistasis subset, $\mathrm{n}=208442$.

\section{Aggregated epistatic interactions predict tertiary structure} contacts

To distill direct contacts from a list of thousands of epistatic double mutants we first aggregated epistatic information on the amino acid position-pair level by calculating the fraction of positive or negative epistatic double mutant variants per position pair (Figure 2A).

In the GB1 epistasis dataset even moderate enrichments for positive and negative epistatic variants are mutually exclusive (Extended Data Figure 3A). Moreover, the strongest positive and negative epistatic enrichments are separated in two clusters of proximal positions in the protein that exhibit mostly either positive or negative interactions among themselves, but hardly any epistatic interactions between clusters (Figures 2B,D and Extended Data Figure 3B), as also noted before by Olson, et al. ${ }^{11}$.

Consistent with epistatic interaction clusters forming a dense network of proximal positions, we find that, of the top 55 epistatic pairs, $42 \%$ and $35 \%$ are direct contacts (connected by one edge smaller than 8 Ångström ( $\AA$ ), 3.9 and 3.2-fold over expectation) and another $45 \%$ and $55 \%$ share a common neighbor (connected via two edges $<8 \AA$ ), for positive and negative epistatic interactions respectively, while interactions across more edges are depleted (Figure 2C; throughout the manuscript we only consider position pairs spaced by more than 5 amino acids in the linear sequence; closer positions are trivially also close in 3D space, and their proximity contributes little to successful structure prediction ${ }^{28}$ ).

While aggregation of epistatic information between position pairs thus better discriminates structural contacts than individual epistatic interactions, positive and negative epistatic interactions still contain disparate structural information of the protein domain. We therefore merged positive and negative epistatic information by computing the weighted averages of 
145 epistatic fractions per position pair given their uncertainty due to fitness measurement errors

146 and the finite number of observed double mutant variants via a resampling approach (Extended

147 Data Figure 1 and Methods). A final epistasis score per position pair was obtained by

148 normalizing these weighted averages by their uncertainty (a z-score), thus giving priority to

149 position pairs with high confidence enrichments.

150 The position pairs with highest epistasis scores are well distributed across the domain (Figures $1512 \mathrm{D}, \mathrm{E}$ ), and the number of direct contacts (one edge $<8 \AA$ ) among the top 55 epistasis score 152 pairs increases to $60 \%$ (Figure 2D), thus showing that the epistasis score successfully 153 incorporates information from both positive and negative epistasis to discriminate direct 154 contacts. Moreover, direct contacts as a whole are enriched for high epistasis scores, while 155 further away position pairs show a gradual decrease of epistasis scores (Pearson correlation 156 coefficient $R=-0.39, p<10^{-6}$, Figure $2 F$ ).

157 Thus, although many interactions are indirect, physical contacts are an important determinant of 158 epistasis and aggregating information on position pairs and merging positive and negative 159 epistasis information better discriminates these direct structural contacts across the protein 160 domain. 
bioRxiv preprint doi: https://doi.org/10.1101/303875; this version posted Auqust 18,2018 . The copyright holder for this preprint (which was not certified by peer review) is the author/funder, who has granted bioRxiv a license to display the preprint in perpetuity. It is made available under aCC-BY-NC-ND 4.0 International license.

Figure 2

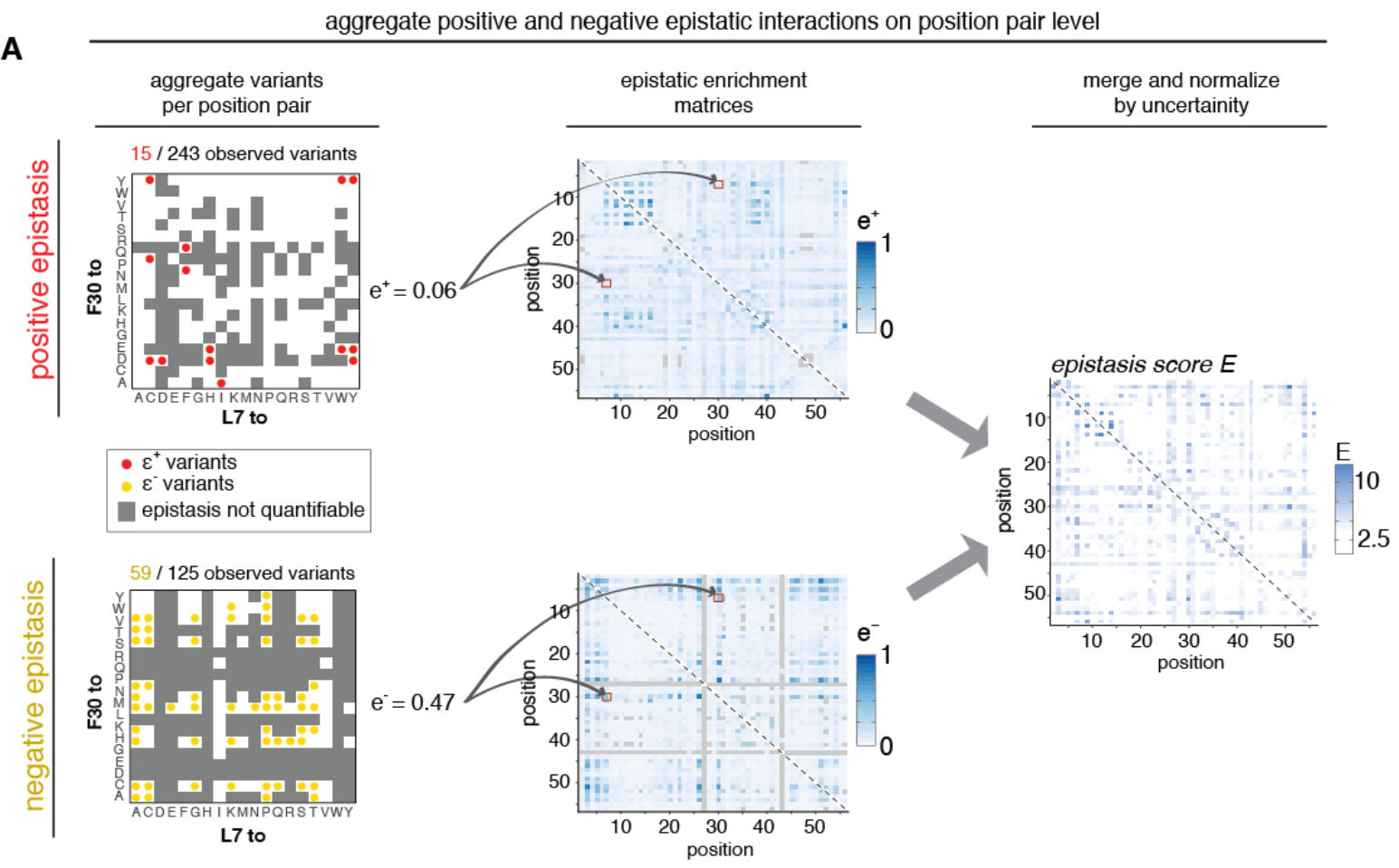

B

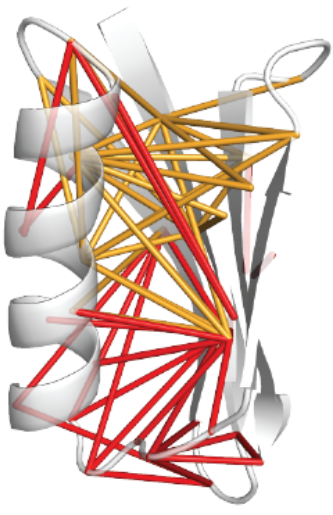

top 28 neg. epistasis pairs top 28 pos. epistasis pairs top 28 epistasis score pairs

E

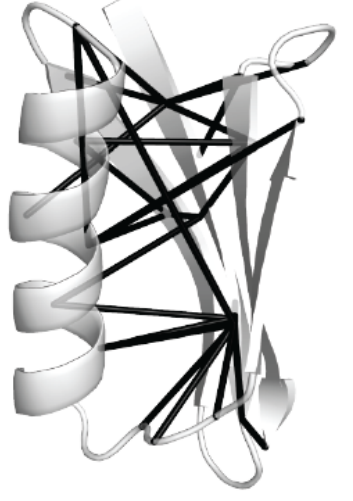

C

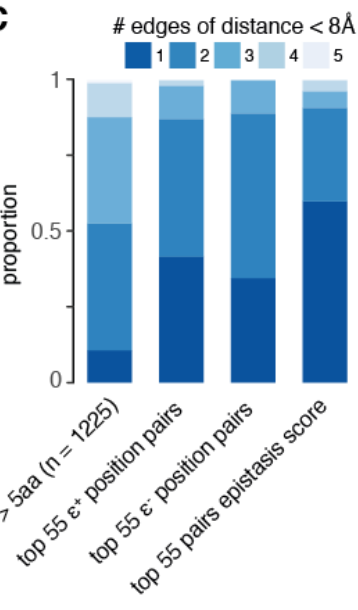

$\mathbf{F}$

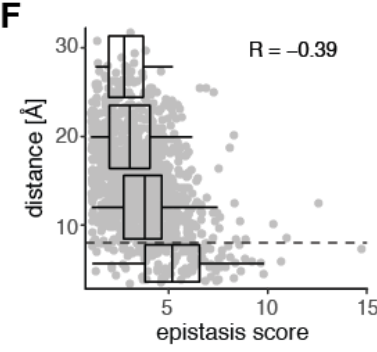

D

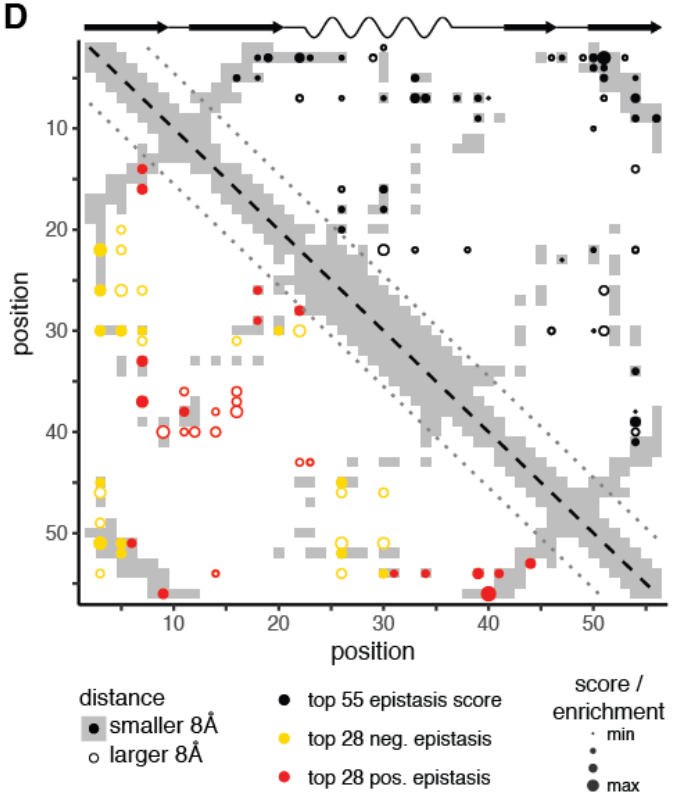


163

A. Workflow for aggregating positive and negative epistatic interactions on the position-pair level and merging them into a final epistasis score.

B. Top 28 position pairs (> 5 amino acids in linear sequence) each with highest positive (red) and negative (yellow) epistatic fractions marked on the reference structure (PDB entry $1 \mathrm{pga})$.

C. Minimal number of edges (contact with distance $<8 \AA$ in reference structure) connecting position pairs. One edge - positions are direct contacts, two edges - positions have a common contact and so forth.

D. Interaction score map for top 28 position pairs each with highest positive (red) and negative (yellow) epistatic fractions (lower left triangle) and top 55 position pairs with highest epistasis score (upper right triangle). Dot size indicates relative epistatic enrichments or score; dot fill indicates distance below $8 \AA$. Underlying in grey is the contact map of the reference structure (PDB entry $1 \mathrm{pga}$, distance $<8 \AA$ ) and shown on top its secondary structure elements (wave - alpha helix, arrow - beta strand).

E. Top 28 position pairs (> 5 amino acids in linear sequence) with highest epistasis scores marked on the crystal structure (PDB entry 1pga).

F. Distance of position pairs as a function of epistasis scores. Boxplots are spaced in distance intervals $[0,8),[8,16),[16,24)$ and $[24,32) \AA$. Dashed horizontal line indicates $8 \AA \AA$. Pearson correlation coefficient is indicated.

\section{Tertiary structure neighborhood leads to correlated epistatic} patterns

If epistasis arises mainly from structural interactions, a position's epistatic interaction profile with all other positions in the protein should provide a signature of its structural location (Figure $3 \mathrm{~A}$ ). Comparing these signatures between positions should thus reveal structurally close positions similar to how correlated epistasis profiles in genetic interaction networks serve to identify physical and functional interaction partners ${ }^{40}$. 
To test the idea that pattern correlation should reveal structural proximity, we calculated the correlations between the epistatic enrichment vectors for all position pairs (Figure 3B). Consistently, pair-distances and similarity of epistasis patterns between positions are strongly correlated (Pearson correlation coefficient $=-0.43, p<10^{-6}, n=1225$, Figure 3D). Top correlated pairs from positive or negative interaction patterns do, however, form mutually exclusive clusters within the protein domain that are nearly identical to the clusters observed for direct positive and negative interactions (Figure $3 \mathrm{C}$ and $\mathrm{E}$, c.f. Figure 2B). Thus, while correlations of epistatic interaction patterns are a good indicator of distance within the protein structure, they suffer from the same issues as epistatic enrichments, namely poor discrimination of direct and indirect interactions and disparate structural information.

We reasoned that partial correlations - the association between two positions after accounting for the global correlation structure - might provide the possibility to eliminate the dependencies observed in the epistasis pattern structure and thus help to distinguish direct from indirect contacts; similar to how mean-field approaches can help discriminate direct from indirect evolutionary couplings in multiple sequence alignments ${ }^{22,28,41}$. We derived partial correlations by inversion of the correlation matrices, merged values from positive and negative epistatic patterns by their estimated uncertainty, and ranked these merged values by their z-scores, which we refer to as association scores (Figure 3B and Methods).

In contrast to the correlation of epistasis patterns, partial correlation of epistasis patterns for both positive and negative epistasis display no clustering but are well distributed across the whole protein domain, consistent with partial correlations removing dependencies between correlated pairs (Figure 3C). Moreover, the merged association scores are less well correlated with pair-distance (Pearson correlation coefficient $R=-0.26, p<10^{-6}, n=1225$ ) and show a more binary all-or-none response, with most distant position pairs having an association score around 0 and only proximal pairs systematically deviating to higher values (Figure 3D). Moreover, the top pairs involve many different individual positions and are well distributed across the protein domain (Figure $3 \mathrm{C}$ and $\mathrm{E}$ ). Thus, association scores are able to prioritize direct over indirect structural contacts across the whole protein domain. 


\section{Combining epistasis and association scores better discriminates 220 structural contacts}

221 We derived a combined score by summing the standardized epistasis and association scores,

222 to explore whether combining information from individual epistatic interactions and epistasis 223 interaction patterns can improve proximity estimates; thereby prioritizing position pairs that are 224 both enriched for direct epistatic interactions and have correlated epistasis patterns.

225 We evaluated the precision of the three interaction scores in predicting direct contacts in the 226 protein domain. For all pairs separated by more than 5 amino acids in the linear sequence, the 227 epistasis score has a roughly constant precision of around $60 \%$ across the first $2 *$ predicted 228 contacts ( $L$ being the mutated length of the protein i.e. 55 amino acids). The association score 229 has higher precision than the epistasis score up to the first $L$ predicted contacts, with a precision 230 of $79 \%$ at L/2 top contacts. Finally, the combined score has similar precision to the association 231 score for the first L/2 contacts, but then remains at higher precision, with an improvement of 232 about $10-15 \%$ over the individual scores at more predicted contacts ( $73 \%$ at L contacts); 233 showing that combining information from epistatic interactions and interaction patterns further 234 improves the discrimination of direct structural contacts.

235 Together, the derivation of the interaction scores demonstrates that it is possible to discriminate 236 direct three-dimensional structural contacts from a mainly non-proximal set of epistatic 237 interactions within a protein domain. 
bioRxiv preprint doi: https://doi.org/10.1101/303875; this version posted August 18, 2018. The copyright holder for this preprint (which was not certified by peer review) is the author/funder, who has granted bioRxiv a license to display the preprint in perpetuity. It is made available under aCC-BY-NC-ND 4.0 International license.

Figure 3

correlation of epistatic patterns

A

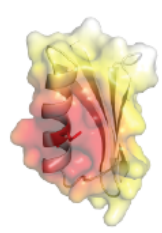

a

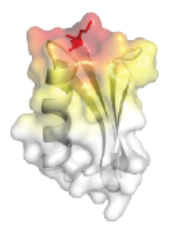

b

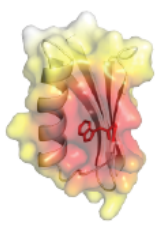

C

$$
\operatorname{corr}\left(\varepsilon_{a, 1, L}, \varepsilon_{c, 1 . L}\right)>\operatorname{corr}\left(\varepsilon_{a, 1 . .}, \varepsilon_{0,1 . . L}\right) \approx \operatorname{corr}\left(\varepsilon_{0,1.1 .}, \varepsilon_{c, 1, \ldots}\right)
$$

chance of epistatic interaction

B

epistatic enrichme matrices
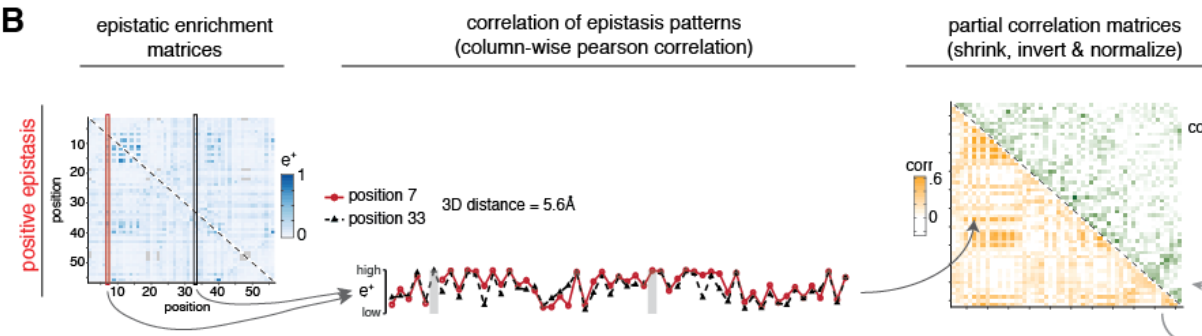

merge positive \& (shrink, invert \& normalize) negative par. correlations

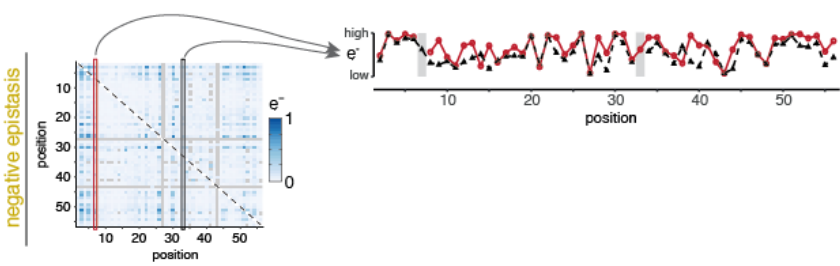

C
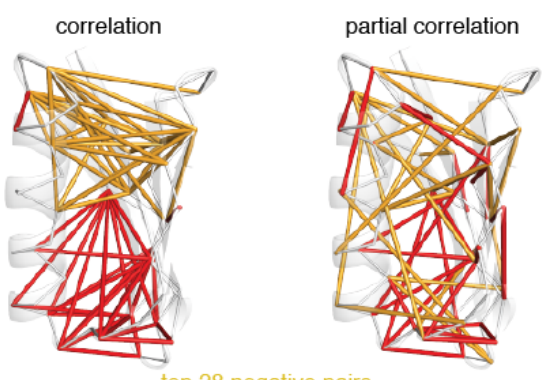

top 28 negative pairs top 28 positive pairs
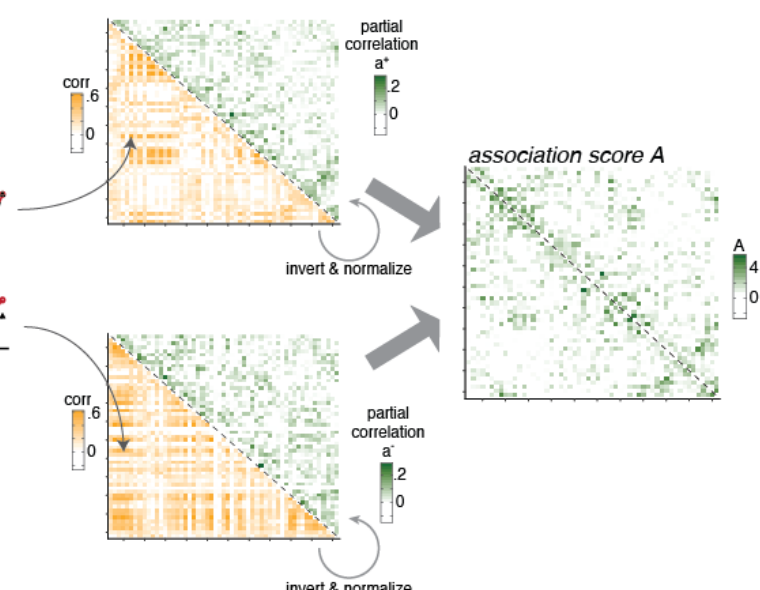

E
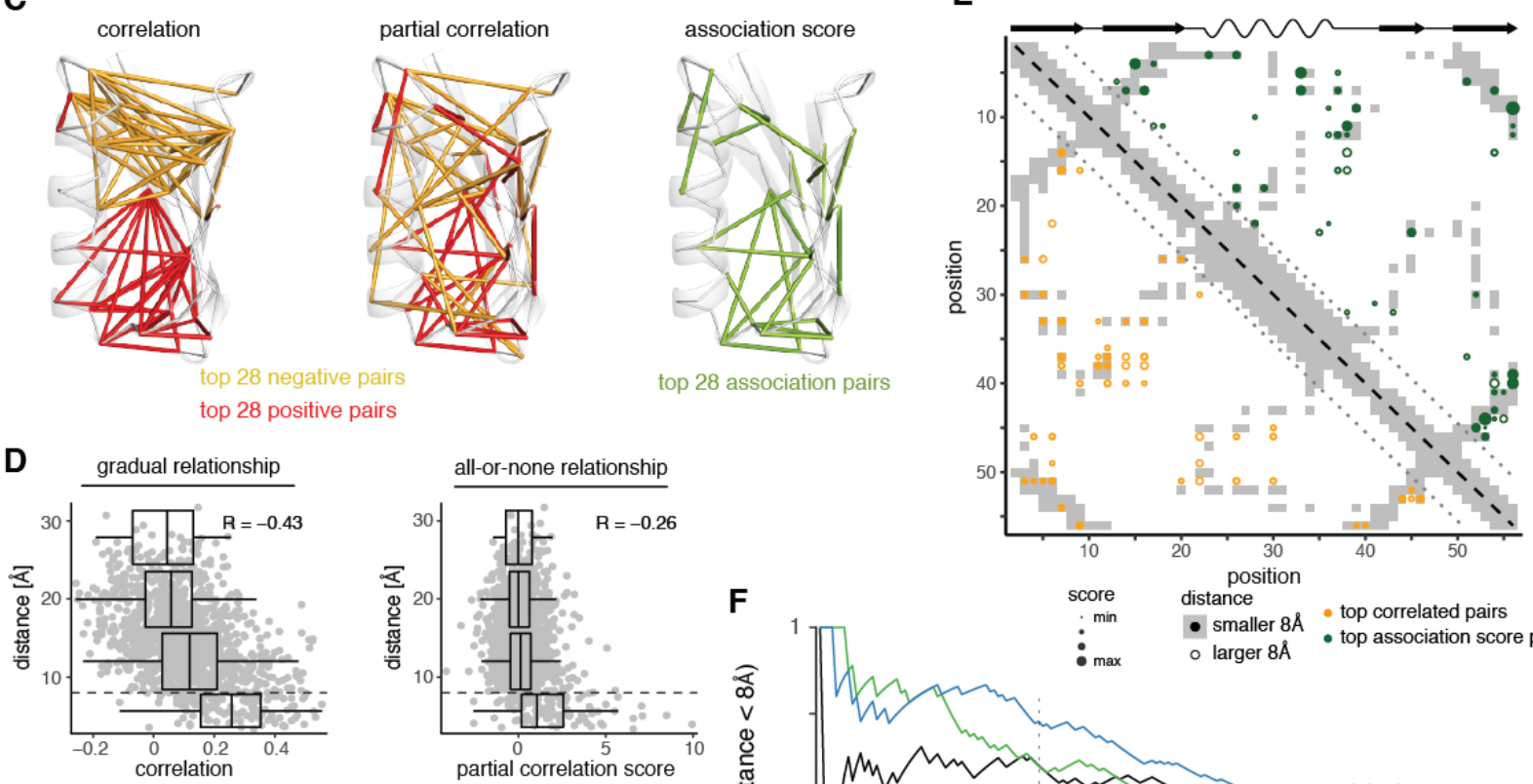

F

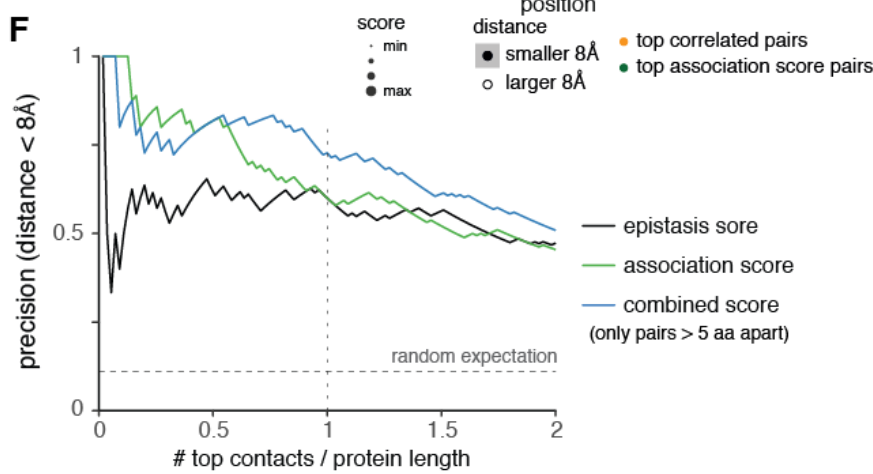


239 Figure 3: Tertiary structure neighborhood leads to correlated epistatic 240 patterns

A. Mutations in directly contacting residues should interact similarly with all other mutations in the protein. Thus, the similarity of epistasis patterns of two positions with all other positions in the protein should inform about their structural proximity.

B. Workflow for quantification of correlated epistasis patterns. Pairs of columns from epistatic enrichment matrices (here columns 7 and 33) are compared and their Pearson correlation coefficients are calculated, which constitute entries in the correlation matrix (here entries 7:33 and 33:7, due to matrix symmetry). Correlation matrices are inverted to yield the partial correlation matrices. Finally, entries of the positive and negative partial correlation matrices are merged (weighted average by uncertainty) and znormalized to yield association scores (see Methods).

C. Top 28 position pairs ( $>5$ amino acids in linear sequence) marked on reference structure. Left: Top pairs from positive (red) or negative (yellow) epistasis pattern correlations. Middle: Top pairs after partial correlation transformation. Right: Top association score pairs (merge positive and negative partial correlations).

D. Distance of position pairs as a function of merged correlation (left) or association scores. Boxplots are spaced in intervals of $8 \AA$. Dashed horizontal line indicates $8 \AA$. Pearson correlation coefficient is indicated.

E. Interaction score map for top 55 position pairs with highest merge correlation (positive and negative correlations merged, lower left triangle, orange) and association scores (upper right triangle, green). Dot size indicates relative correlations or scores; dot fill indicates distance below $8 \AA$. Underlying in grey is the contact map of the reference structure (PDB entry 1pga, distance $<8 \AA$ ) and shown on top its secondary structure elements (wave - alpha helix, arrow - beta strand).

F. Precision of interaction scores to predict direct contacts (distance $<8 \AA$ in crystal structure 1pga) as a function of top scoring position pairs. Only position pairs with linear chain distance greater than 5 amino acids are considered $(n=1225$ pairs, $n=131$ direct contacts in reference structure). Horizontal dashed line indicates random expectation. 


\section{Periodic epistatic patterns reveal secondary structure}

\section{9 arrangements}

270 We investigated whether the periodic geometrical arrangement of amino acid residues in 271 secondary structures results in periodic epistasis patterns ${ }^{26,42}$. Within an alpha helix with 3.6

272 residues per helical turn, a helical position would be predicted to interact epistatically with the

273 third or fourth-over position along the linear amino acid chain (Figure 4A). Equivalently, within a

274 beta strand, positions should interact epistatically with the next-but-one position.

275 We used a two-dimensional kernel smoothing approach to estimate the positions of alpha 276 helices and beta strands from the deep mutational scanning data (Figure 4B). Here, the 277 propensity of a position to belong to an alpha helix or a beta strand depends on whether it 278 shows the expected periodicity in its interaction with neighboring positions, as well as whether 279 neighboring positions display similar propensities for the same secondary structure element, 280 and how strong these interactions are compared to those found in randomized data sets (see 281 Methods).

282 We found that, while secondary structure element predictions derived from direct interaction283 based epistasis scores are somewhat inaccurate and underpowered, predictions derived from 284 correlation-based association scores (as well as combined scores) coincide very well with 285 secondary structure elements in the reference structure (Figure 4C and Extended Data Figure $2864 \mathrm{C}$ ), with precision and recall values of about 90\% (Extended Data Figure 4D). This suggests 287 that the correlated profiles of epistatic interactions are informative about side chain orientations 288 and also that eliminating transitive interactions is important for (secondary) structure prediction.

289 We further used two-dimensional kernel smoothing to detect parallel and anti-parallel beta sheet 290 interactions, by applying beta strand kernels to off-diagonal entries on the interaction score 291 matrices (Extended Data Figure 4A, see Methods). Several stretches of position pairs show the 292 expected alternating interaction profiles for either parallel or anti-parallel beta sheets (Extended 293 Data Figure 4B), with the top predictions corresponding to the known beta-sheet interactions in 294 the reference structure (Figure 4D). Furthermore, updating beta strand predictions according to 295 inferred beta sheet pairings can further improved beta strand prediction itself, notably 296 introducing a correct split of beta strand 1 and 2 and adjusting the length of beta strands 3 and 4 297 (Figure 4C,D). 
bioRxiv preprint doi: $\mathrm{https}$ //doi.org/10.1101/303875; this version posted August 18 , 2018. The copyright holder for this preprint (which was not certified by peer review) is the author/funder, who has granted bioRxiv a license to display the preprint in perpetuity. It is made available under aCC-BY-NC-ND 4.0 International license.

Together this shows that epistatic interaction data contains information on the periodic secondary structure of a protein domain and, vice versa, that secondary structure strongly influences genetic interactions.

\section{Figure 4}

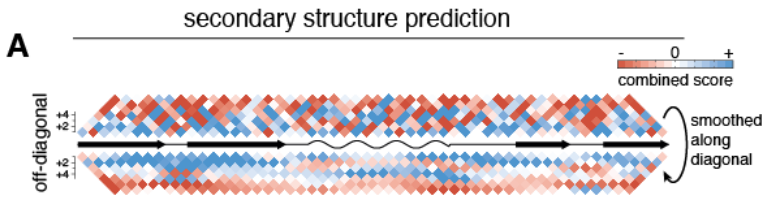

B

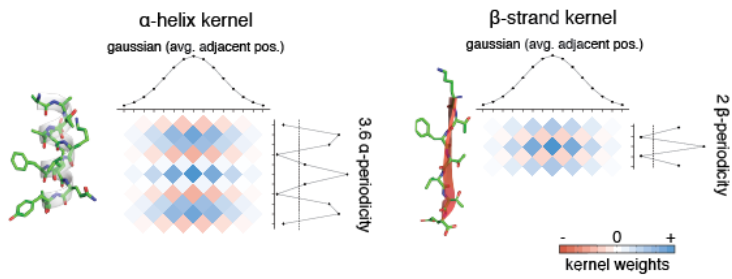

C

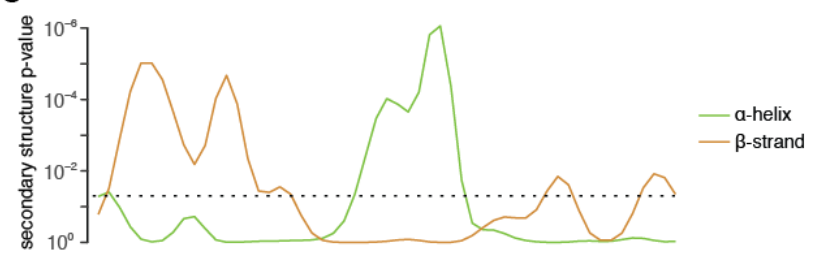

D
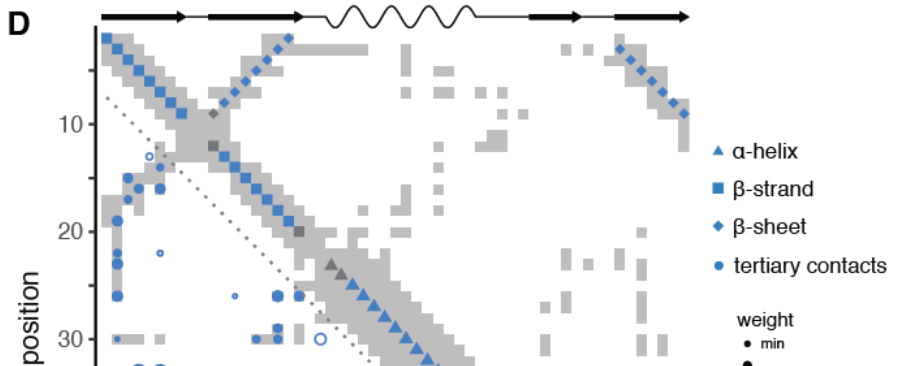

301

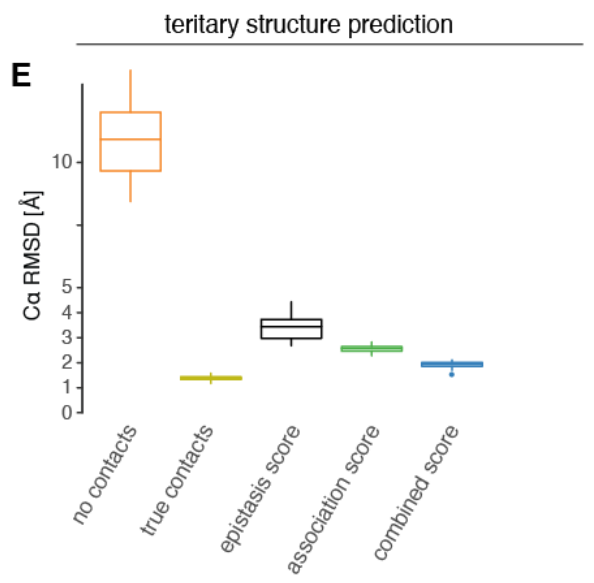

$\mathbf{F}$

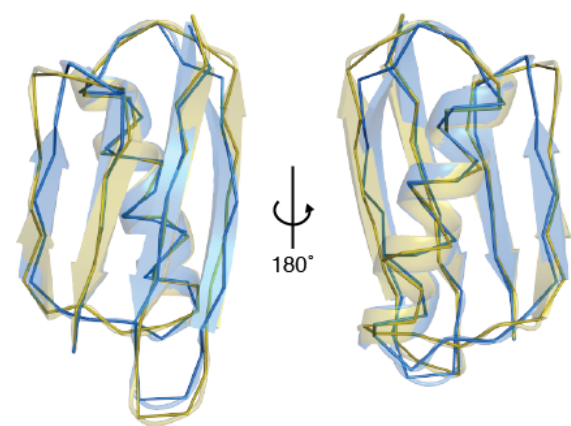

1pga crystal structure

best combined score model (1.5Å Ca-RMSD) 


\section{Figure 4: Secondary and tertiary structure prediction from deep mutational}

\section{3 scanning data}

A. Local interactions reveal signatures of secondary structure elements. Middle line is diagonal of interaction score map (rotated by 45 degree) and shows secondary structure elements of reference structure (PDB entry 1pga). Data above diagonal shows combined score data close to the diagonal, i.e. local interactions. Below the diagonal, the same data are smoothed with a Gaussian kernel along the direction of the diagonal (i.e. horizontally, length of Gaussian kernel as for kernels in panel b) to reveal periodicities in local interactions.

B. Two-dimensional kernels for alpha helix and beta strand detection. Kernel has a sinusoidal or alternating profile in the off-diagonal direction to detect alpha helices and beta strands propensities, respectively and a Gaussian profile along the diagonal, to average over propensities of adjacent positions.

C. Secondary structure propensity derived from kernel smoothing (orange - beta strand, green - alpha helix). P-values were derived by comparison to randomized datasets (see Methods). Dashed line indicates $p=0.05$.

D. All structural predictions derived from combined score data. Lower left: Top 55 non-local (>5 aa in linear sequence) position pairs, i.e. tertiary contacts (circles); fill indicates correct prediction at $8 \AA$, size of circles indicates relative score. Upper right: Predicted secondary structure elements (triangle - alpha helix, square - beta strand, diamond beta sheet interaction). Fill indicates correct prediction. Note that beta strand predictions are derived by intersection of beta strand propensity (as shown in panel C) and results from beta sheet prediction (Extended Data Figure 4B, see Methods). Underlying in grey is the contact map of the reference structure (PDB entry $1 \mathrm{pga}$, distance $<8 \AA$ ) and shown on top are its secondary structure elements (wave - alpha helix, arrow - beta strand).

E. Accuracy ( $C \alpha$ root-mean-square deviation) of top 5\% structural models generated from deep mutational scanning data derived restraints compared to GB1 reference structure. Structural models were generated in XPLOR-NIH by simulated annealing with restraints derived from top 55 top scoring position pairs, secondary structure element prediction and beta sheet pairing predictions from the indicated interaction scores. No contacts negative control with restraints only for secondary structure (predicted by PSIPRED) $)^{43}$. 
True contacts - positive control with 55 contacts (random subset), secondary structure elements and beta sheet interactions restraints derived from reference structure.

\section{Protein structure determination by deep mutagenesis}

341 Together, these findings show that deep mutagenesis data contain substantial information about

342 a protein's secondary and tertiary structure. We therefore tested whether the data would suffice

343 to determine $a b$ initio the structure of protein $\mathrm{G}$ domain $\mathrm{B} 1$. We performed structural simulations by simulated annealing using the XPLOR-NIH modeling suite ${ }^{45}$, with structural restraints derived from the deep mutational scanning data (see Methods). In particular, we defined distance restraints (distance $<8 \AA$ between $C \beta$ atoms) for the top scoring position pairs; we found that using the top $L(L=55)$ contacts gave best results (Extended Data Figure 4F). Furthermore, we defined dihedral angle restraints for predicted secondary structure elements. Finally, we defined restrictive distance restraints (distance smaller than $2.1 \AA$ for $\mathrm{N}-\mathrm{H}: \mathrm{C}=\mathrm{O}$ atom pairs) for beta sheet positions that form hydrogen bonds with each other.

We evaluated the top $5 \%$ of structural models (25/500, evaluation based on XPLOR internal energy terms) generated against the known crystal structure of protein $G$ domain B1 (PDB entry 1pga) (Figures 4E and Extended Data Figure 4F). Models predicted from combined score data performed best, with an average Ca-root mean squared deviation of the top models ( $\langle C \alpha-$ $R M S D\rangle)$ of $1.9 \AA$ and an average template modeling score of 0.71 , which is very close to the optimum achievable with our simulation protocol (using contacts, secondary structure elements and beta sheet interactions from the reference structure, $\langle C \alpha-R M S D\rangle=1.4 \AA$ and $\mathrm{TM}$ score $=$ 0.8 ); and the top evaluated combined score structural model has a $C \alpha-R M S D$ of only $1.5 \AA$ (Figure 4F). Consistent with somewhat lower precision of contact and secondary structure predictions, models generated with restrains from epistasis or association scores have on average a lower accuracy $(\langle C \alpha-R M S D\rangle=3.4 \AA$ and $\langle C \alpha-R M S D\rangle=2.6 \AA$, respectively), with association score models performing consistently better (Figures 4E and Extended Data 4F). structure of a protein domain. 


\section{Contact prediction in additional protein domains}

366 To test the generality of our approach, we analyzed two additional, incomplete deep mutational 367 scanning datasets. First, a mutational scan of the 75 amino acid Pab1 RRM2 domain (Figure $3685 \mathrm{~A}$ ), for which fitness was assessed in a complementation assay ${ }^{10}$. Second, a mutational scan 369 of the hYAP65 WW domain (Figure 5C), in which 33 out of 50 amino acids were mutated and 370 fitness was assayed by binding to a polyproline peptide ligand in a phage display assay ${ }^{46}$. Both 371 datasets were created by 'doped' oligonucleotide synthesis and thus consist primarily of amino 372 acid changes elicited by just one nucleotide change, which results in only $10 \%$ of possible 373 double mutants being present. Additionally, their selection assays have smaller measurement 374 ranges than that of the GB1 domain, which results in higher relative errors of fitness estimates 375 as well as in negative epistasis being quantifiable for a smaller fraction of double mutants, as 376 low as $0.8 \%$ in the case of the WW domain (Extended Data Figure 5A, see Table 1 for 377 comparison of dataset properties).

378 For the RRM domain, three 25 amino acid segments were mutated separately and we restricted our analysis to the central one, as it is the only segment that exhibits a reasonable number of intra-segment contacts in the reference structure (Figure 5A). We find that predicted tertiary contacts fall on or very close to known contacts in the region of the anti-parallel beta sheet and the intervening loop region (Figure 5B), with a precision of $57 \%$ for the top $L / 2$ and $50 \%$ for the top $L$ position pairs of the combined score (3-fold and 2.7-fold over expectation, respectively). Predicted beta strand propensities peak at the correct positions, albeit with low statistical significance; additionally, an alpha helical propensity is detected in the intervening loop region. Nonetheless, the correct anti-parallel beta sheet conformation at the correct position pairs is predicted.

For the WW domain, we find that top predicted tertiary contacts fall on or very close to known interactions between the beta strands and the $\mathrm{N}$-terminal and C-terminal loop regions (Figure $5 D$ ), with a precision of $59 \%$ for the top $L / 2$ and $38 \%$ for the top $L$ position pairs of the combined score (3.9-fold and 2.5-fold over expectation, respectively). Secondary structure elements are 392 not well predicted, but beta sheet interactions are predicted in the right anti-parallel 393 conformation of $\beta 1-\beta 2-\beta 3$, though the exact pairing between positions is off by one to two 394 positions. We determined the three dimensional structure of the secondary structure-rich central 395 part of the domain (positions 6 to 29, 24 amino acids), using restraints derived from top 396 combined score pairs and PSIPRED-predicted secondary structure elements (see Methods). 
397 The top $5 \%$ of structural models have an average accuracy of $3.3 \AA\langle C \alpha-R M S D\rangle$ compared to 398 the reference structure, which is on par with simulations using a set of 'true' contacts $(\langle C \alpha-$ $399 R M S D\rangle=3.6 \AA$ ) (Extended Data Figure $5 \mathrm{C}$ ). Moreover, the structural model with the best 400 XPLOR-NIH energy has an accuracy of $2.4 \AA C \alpha-R M S D$ (or $2.0 \AA$ over 22 of the 24 residues) 401 (Figure 5C). Despite similar precision of predicted contacts, association and combined score402 derived WW domain structural models are more accurate than epistasis score-derived models 403 (Extended Data Figure 5C).

404 Together these results strongly support the generality of our approach for extracting structural 405 information from deep mutagenesis data, including from sparser and lower quality data. 
bioRxiv preprint doi: https://doi.org/10.1101/303875; this version posted Auqust 18, 2018. The copyright holder for this preprint (which was not certified by peer review) is the author/funder, who has granted bioRxiv a license to display the preprint in perpetuity. It is made available under aCC-BY-NC-ND 4.0 International license.

\section{Figure 5}

A Pab1 RRM2 domain (25/75 aa analysed)
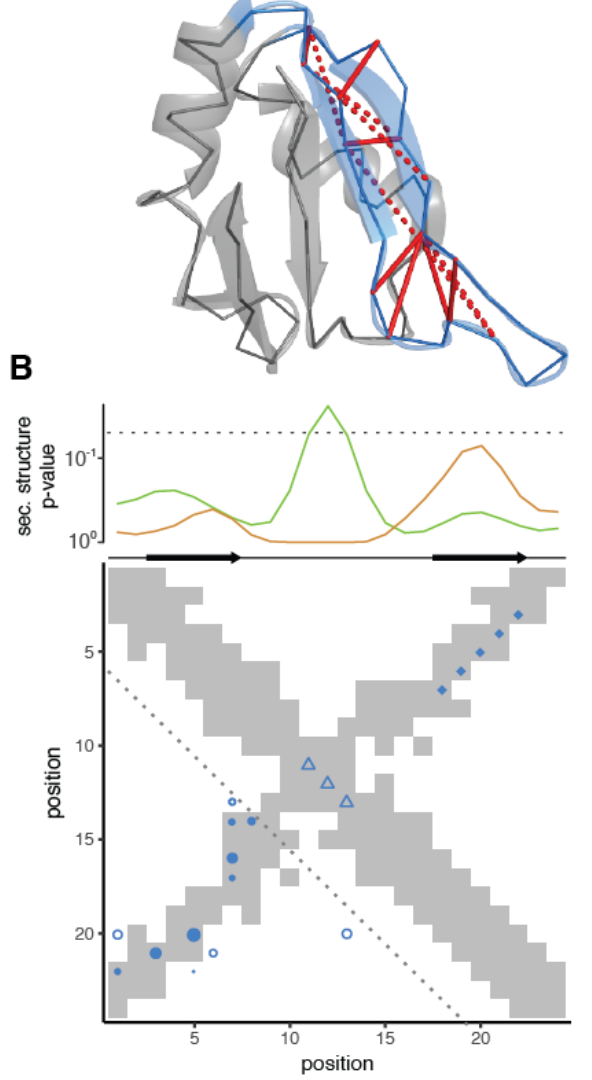

C

hYAP65 WW domain (33/50 aa mutated)

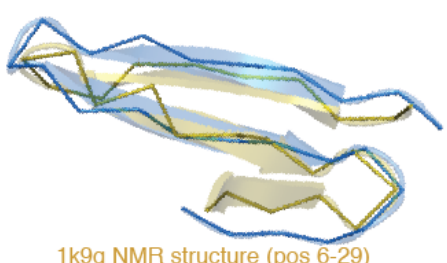

best combined score model (2.4Å Ca-RMSD)

D

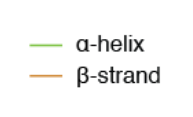

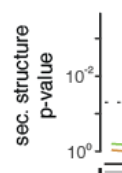

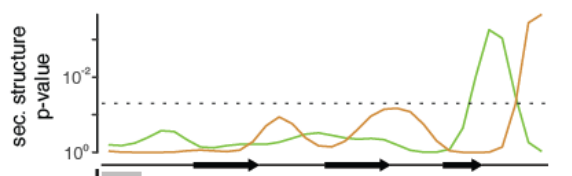

$\Delta$ a-helix

- $\beta$-strand

- $\beta$-sheet

combined score

top contacts

weight

min

:

- max

distance $<8 \AA$

- FALSE

- true

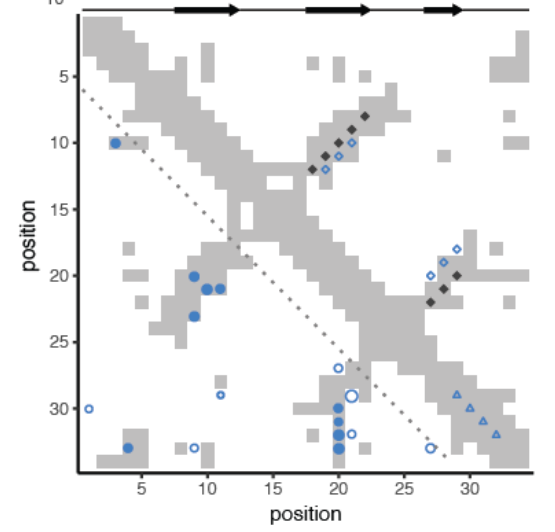

Determining protein-protein interaction contacts and underlying structures

\section{E}

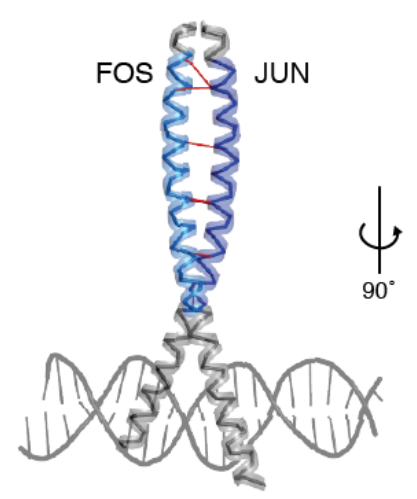

$\mathbf{F}$

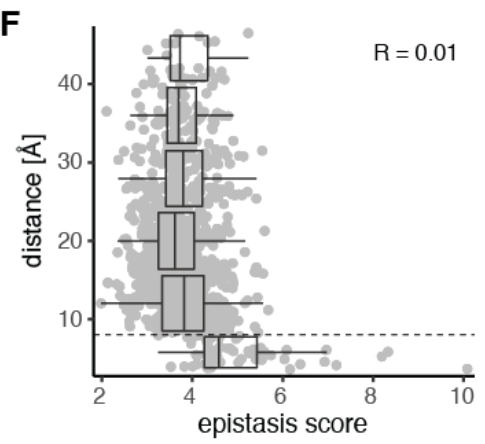
G secondary struct. propensity (from assocation scores)
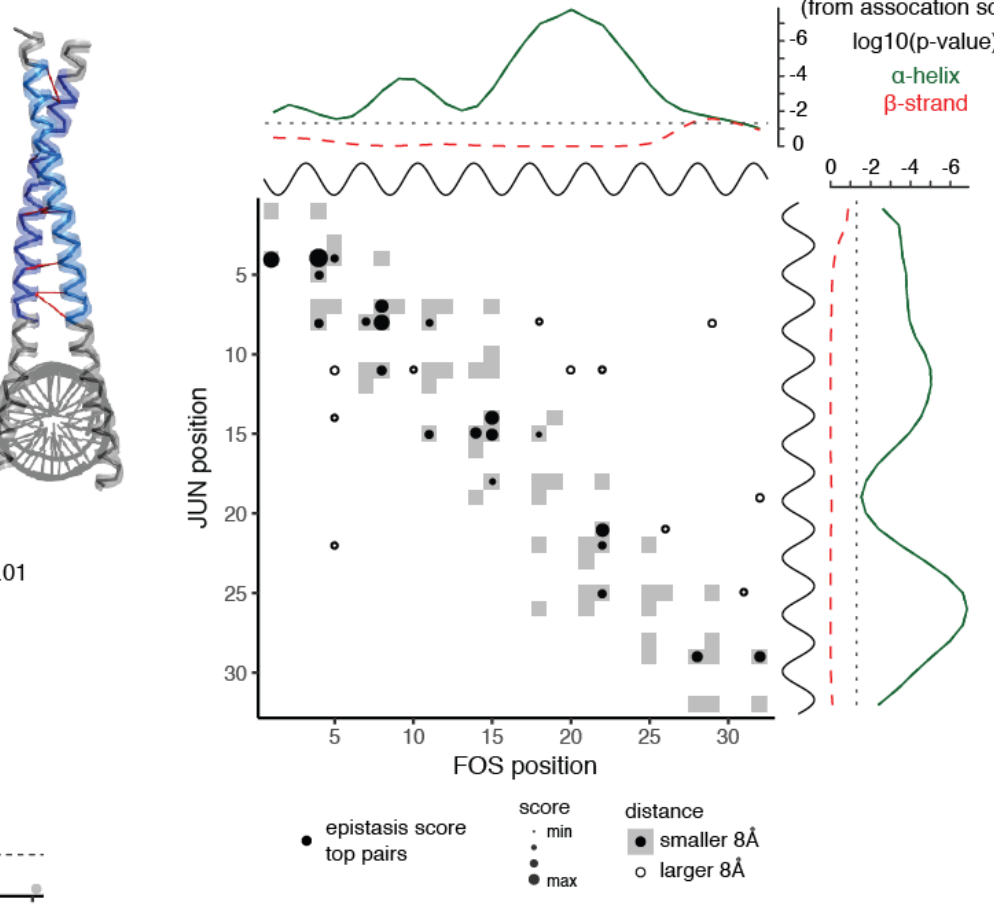
407 Figure 5 - Predicting structural contacts in two additional proteins and a 408 protein-protein interaction

A. Pab1 RRM2 domain structure (PDB entry 1cvj) with 25/75 positions analyzed here highlighted in blue. Top 12 combined score position pairs are connected with red lines, solid if distance $<8 \AA$, dashed otherwise.

B. Structural predictions derived from combined scores in RRM domain. Upper plot shows secondary structure propensities from kernel smoothing $(p=0.05$ indicated as dashed line). Just below are shown the secondary structure elements in the reference structure. Map shows top 12 combined score position pairs in lower left and secondary structure predictions in upper right triangle. Shape indicates type of prediction, fill indicates correct prediction. Underlying is the contact map of the reference structure (grey if $<8 \AA$ ).

C. Overlay of top structural model of hYAP65 WW domain (positions 6-29) generated with restraints from combined score (blue) and solution NMR structure (gold, PDB entry $1 \mathrm{kgq})$.

D. Structural predictions derived from combined scores in WW domain. Upper plot shows secondary structure propensities from kernel smoothing $(p=0.05$ indicated as dashed line). Just below are shown the secondary structure elements in the reference structure. Map shows top 17 combined score position pairs in lower left and secondary structure predictions in upper right triangle. Shape indicates type of prediction, fill indicates correct prediction. Underlying is the contact map of the reference structure. Black diamonds indicate positions of beta sheet pairing in reference structure. Crystal structure of the leucine zipper domains of FOS and JUN with a DNA strand (PDB entry 1fos). The mutated regions (32 amino acids each) are highlighted in light blue (FOS) and dark blue (JUN). Top 10 epistasis score pairs are shown with red dashes.

E. Distance of position pairs as a function of interaction scores. Boxplots are spaced in distance intervals of $8 \AA$. Dashed horizontal line indicates $8 \AA$. Pearson correlation coefficient is indicated.

F. FOS-JUN trans interaction score map for top 32 position pairs with highest epistasis scores. Note that protein-protein interaction maps are not symmetric. Dot size indicates relative score; dot fill indicates distance below $8 \AA$; underlying in grey is the contact map of the reference structure (PDB entry 1 fos, distance $<8 \AA$ ). Shown on top and to the right 
of the contact map are the known alpha helices and secondary structure propensities derived from association scores of FOS and JUN, respectively (black - known alpha helix; green - predicted alpha helix propensity, orange - predicted beta strand propensity; see Extended Data Figures 5F,G).

\section{Contact prediction in a protein-protein interaction}

Genetic interactions do not only occur between mutations within individual proteins but also between molecules that physically interact ${ }^{2}$. We investigated a deep mutational scanning dataset of the coiled-coil interaction between the proteins encoded by the proto-oncogenes FOS and JUN (Figure 5E) ${ }^{9}$. In this experiment, all possible single amino acid changes were made in each of 32 positions of each protein and the physical interaction of all single and (trans-)double mutants was quantified using a deep sequencing-based protein complementation assay. After filtering, the dataset contains $43 \%$ of all possible double mutants and has a median relative error of fitness measurements of $3.6 \%$ (Table 1 ).

When assessing the enrichment of epistatic interactions between positions in the two interaction partners we find a striking all-or-none relationship between epistasis scores and pair-distances (Figure 5F), with all distant pairs contained in a low epistasis score peak and only proximal interactions enriched for high epistasis scores (Pearson correlation coefficient $\mathrm{R}=0.01, \mathrm{n}=$ 1024). Indeed, the top 11 epistasis score pairs are all proximal interactions, and the precision of contact prediction is $75 \%$ for the top $L / 2$ contacts and $66 \%$ for the top $L$ contacts (12-fold and 10.5-fold over expectation). Moreover, top epistasis score pairs are evenly distributed across the interaction surface (Figures $5 \mathrm{E}$ and $5 \mathrm{G}$ ).

When correlating epistatic patterns between columns of the epistatic enrichment matrices, one is comparing the epistatic interactions that two positions in FOS have with all positions in JUN. Therefore, the similarity of column-wise epistatic patterns reveals the cis relationships between positions in FOS (Extended Data Figure 5F). Similarly, correlating epistasis patterns across combinations of rows of the epistatic enrichment matrices reveals cis relationships between positions in JUN. We find that cis-interaction maps from association scores for both FOS and JUN are highly enriched for strong local interactions with an alpha helical periodicity; and applying our secondary structure prediction algorithms to the cis-interaction maps reveals strong alpha helix propensities across the full lengths of both FOS and JUN (Figures 5G and Extended Data Figure 5G). 
This shows that deep mutagenesis of protein interaction partners can accurately predict direct contact across the interaction surface as well as the underlying structures of the interaction partners themselves.

474 Evolutionary coupling-based structural predictions have been successfully improved by machine 475 learning approaches that transform the two-dimensional interaction score maps after learning 476 the stereotypical patterns between evolutionary coupling-predicted contact maps and the actual contact maps of the known structures ${ }^{47,48}$.

478 We tested whether such an approach can also improve deep mutagenesis-derived contact 479 predictions. We applied a convolutional neural network approach called DeepContact, 480 developed by Liu, et al. ${ }^{47}$. The basic DeepContact architecture takes as a sole input a two481 dimensional interaction score map that it then transforms based on the structural patterns it has previously learned on evolutionary coupling-derived contact predictions for representative families of the SCOPe database ${ }^{49}$ (Figure 6A and Methods). When transforming evolutionary coupling-derived contact predictions of proteins not contained in the training set, this basic DeepContact architecture has been shown to improve contact prediction precision by about 10$20 \%{ }^{47}$.

487 We first transformed the GB1 domain combined score interaction map with the DeepContact network (Figure 6B). These transformations take as sole input our deep mutational scanningderived predictions and include no evolutionary coupling or otherwise-derived structural predictors for GB1. The scores on the transformed map are much less noisy, with high scores exclusively focused in areas of known contacts, especially those of secondary structure element interactions, and areas devoid of contacts showing homogenously low scores. Moreover, the 493 transformed scores are highly correlated with pair-distances in the reference structure (Pearson 494 correlation coefficient $R=-0.68, p<10^{-6}, n=1225$, Extended Data Figure 6A). The precision of 495 top predicted contacts improves from $82 \%$ to $96 \%$ for $L / 2$ and from $73 \%$ to $87 \%$ for $L$ predicted 496 contacts (Figure 6F). Epistasis score-derived predictions improve by about 5\%, while 497 association score-derived predictions improve by $29 \%$ at $L$ predicted contacts. In contrast, 498 randomized interaction score maps show no changes in prediction performance over random 499 expectation after transformation with DeepContact. 
bioRxiv preprint doi: https://doi.org/10.1101/303875; this version posted August 18,2018 . The copyright holder for this preprint (which was not certified by peer review) is the author/funder, who has granted bioRxiv a license to display the preprint in perpetuity. It is made available under aCC-BY-NC-ND 4.0 International license.

Interaction score maps for the other datasets show similar improvements to GB1 both in terms of cleaner interaction score maps that resemble the reference contact maps as well as increases in contact prediction precision of up to $30 \%$ (Figure $6 \mathrm{C}-\mathrm{F}$ ).

503 This shows that machine learning can substantially improve contact map prediction from deep

504 mutational scanning data, allowing the use of sparser and lower quality data for accurate 505 prediction.

506

\section{Figure 6}

A

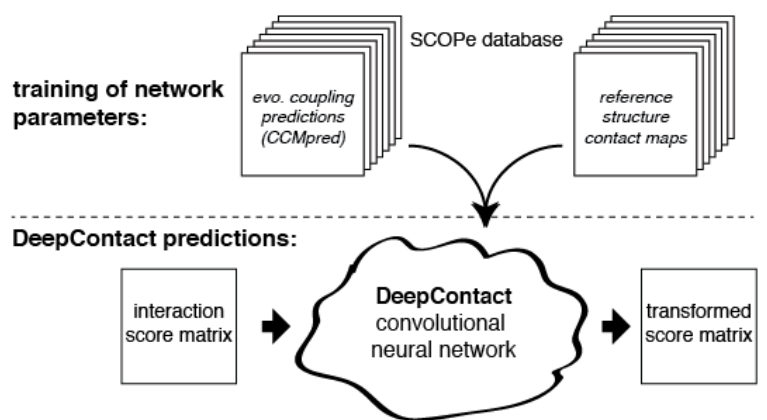

B GB1 domain

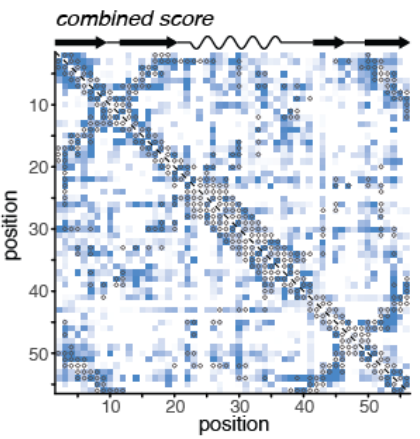

C WW domain (positions 1-29)

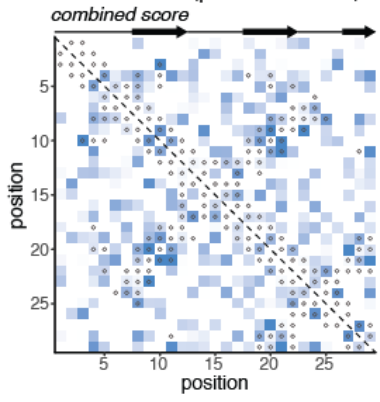

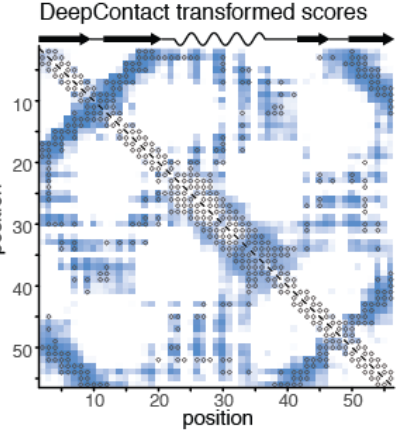

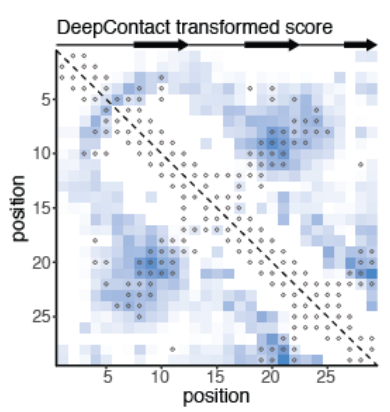

$\mathbf{F}$

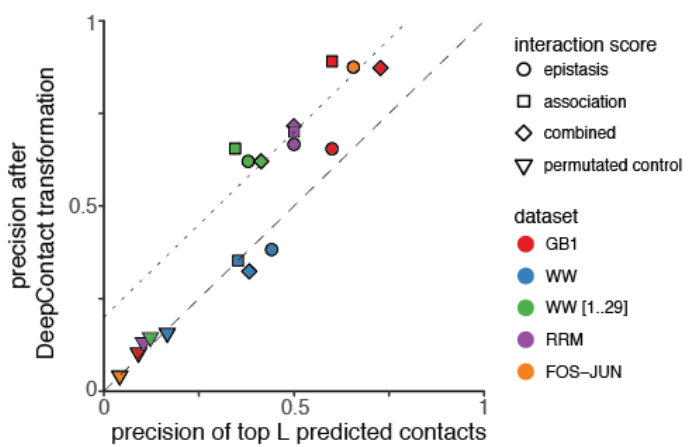

D RRM domain

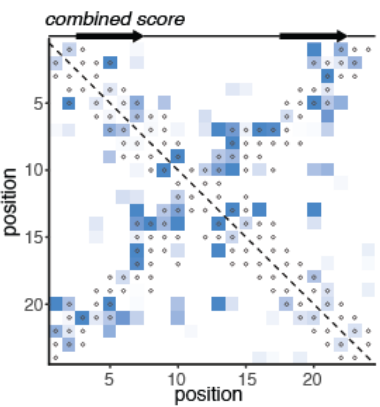

E FOS-JUN interaction
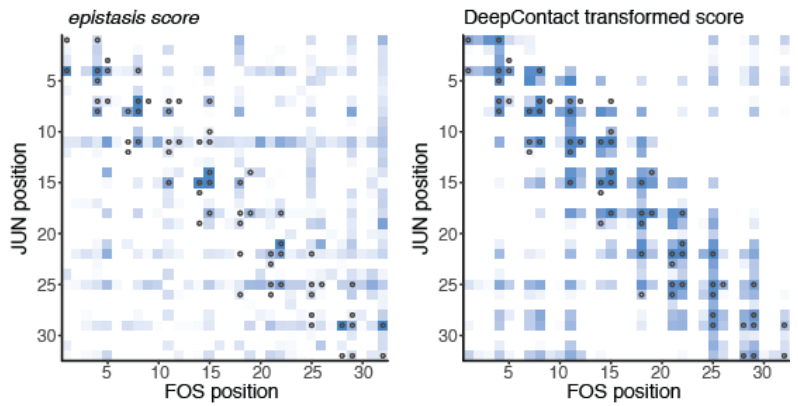
508 Figure 6: Deep learning improves contact prediction from deep

509 mutagenesis data

A. DeepContact convolutional neural network transforms DMS-derived interaction score maps based on learned structural patterns ${ }^{47}$. The particular DeepContact architecture used here takes as only input the DMS-derived interaction score map and transforms it based on structural patterns previously learned on an orthogonal and independent training set (in which it compared evolutionary coupling-derived contact predictions with contacts in known structures of representative protein families in the SCOPe database).

B. GB1 domain combined score interaction map before (left panel) and after (right panel) transformation with DeepContact convolutional neural network. Heat maps show scores (normalized to have similar range). Grey open circles show contacts (side-chain heavy atom distance $<8 \AA$ ) in reference structure.

C. WW domain combined score interaction map before (left) and after (right) DeepContact transformation. Note that the maps shown here lack the $5 \mathrm{c}$-terminal positions (see Extended Data Figure 6B for full map).

D. RRM domain combined score interaction map before (left) and after (right) DeepContact transformation.

E. FOS-JUN trans-interaction epistasis score interaction map before (left) and after (right) DeepContact transformation.

F. Precision of top $L$ predicted contacts of different interaction scores before and after DeepContact transformation for the four datasets. Color indicates dataset, shape indicates interaction score. Permutated control score is average over three random permutations of combined score matrices (in case of FOS-JUN epistasis score matrices). Dashed diagonal line indicates no changes in precision, dotted diagonal line shows precision improvement of $20 \%$ after DeepContact learning. 
 \\ Minimal data quality requirements for successful protein structure} prediction

537 We further investigated how robust our prediction strategy is to changes in data quality by 538 artificially down-sampling the GB1 domain dataset, thus assessing the minimal requirements for 539 deep mutational scanning datasets to be useful for structure prediction.

540 First, we considered the sequencing read coverage. The GB1 domain dataset consists of about 541600 million sequencing reads ${ }^{11}$. We find that artificially down-sampling the sequencing read 542 coverage of the dataset to $25 \%$ or $10 \%$ hardly affects the precision of predicted tertiary contacts 543 (Figures $7 \mathrm{~A}$ ). Only when using just $2.5 \%$ of sequencing reads (15 million) does the precision of 544 top L contacts drop below $50 \%$.

545 Next, we simulated a 'doped' dataset, by only considering amino acid mutations that can be 546 reached by one mutation in the nucleotide sequence - thus reducing the coverage of double 547 mutants to $\sim 10 \%$ (similar to RRM and WW domain datasets). The doped dataset with full 548 sequencing read coverage exhibits a drop in precision of predicted tertiary contacts of about $54920 \%$. Moreover, the doped dataset shows an increased sensitivity to lower sequencing read 550 coverage.

551 We also tested the effect of reducing the signal-to-noise ratio (i.e. the measurement range of 552 selection assay relative to the median error of fitness estimates), which results in non553 quantifiably of negative epistasis (Extended Data Figures 2D-F and 5A). We thus tested how 554 our prediction strategy performs on the GB1 domain dataset when only positive epistasis 555 information is available; and find that it results in a drop of precision of about $20 \%$, comparable 556 to that observed for a doped dataset. In contrast, only using negative epistasis information 557 resulted in a drop to $\sim 35 \%$ precision, as low as a doped dataset with $10 \%$ sequencing 558 coverage.

559 We evaluated secondary structure prediction performance of the various down-sampled GB1 560 domain datasets. Beta strand and alpha helix predictions are hardly affected by lowered data 561 quality or partial epistasis information (Extended Data Figure 7A). In contrast, precision and 562 recall of beta sheet positional pairing is strongly affected by dataset quality, although often the 563 correct overall conformation of beta sheets is still recovered (Extended Data Figure 7B).

564 We next tested whether DeepContact could also improve prediction performance on these 565 down-sampled datasets. Similar to interaction scores derived from full datasets, DeepContact 
transformation of combined scores derived from down-sampled GB1 datasets improves the precision of predicted contacts by about $10-25 \%$ even for quite low quality datasets, i.e. the complete datasets with at least $10 \%$ read coverage, doped datasets with at least $25 \%$ read coverage or the dataset with only positive epistasis information (Figure 7A).

Finally, we evaluated how differences in prediction performance of tertiary contacts affect structural modeling. We find that changes in accuracy of the top structural models roughly scale with changes in contact prediction performance (Figure 7B). Down-sampling of sequencing reads in the complete dataset from $100 \%$ to $2.5 \%$ leads to a drop in accuracy from $2.5 \AA$ to $4 \AA$

$574\langle C \alpha-R M S D\rangle$, which is roughly also the accuracy of top structural models from the doped 575 dataset and the dataset using only positive epistasis information. Accuracies for lower quality 576 datasets range from $5 \AA$ to $9 \AA\langle C \alpha-R M S D\rangle$. DeepContact increases the accuracy of the top 577 structural models by up to $2.6 \AA$. For the complete datasets with only $25 \%$ or $10 \%$ of sequencing 578 reads, the top structural models have better accuracy than those from the complete dataset with 579 full sequencing read coverage but untransformed scores. Also, structural models based on 580 DeepContact transformed scores from the doped dataset with full or $25 \%$ sequencing coverage and those from the dataset using only positive epistasis information reach average accuracies

582 of $3.2 \AA\langle C \alpha-R M S D\rangle$. Only for the two datasets with $2.5 \%$ sequencing read coverage do 583 structural simulations based on DeepContact transformed scores not improve model accuracy.

584 Together these findings show that contact and structure prediction from deep mutational 585 scanning data can also work for lower quality datasets and that the use of deep learning allows 586 the use of much sparser and lower quality datasets.

\section{Figure 7}
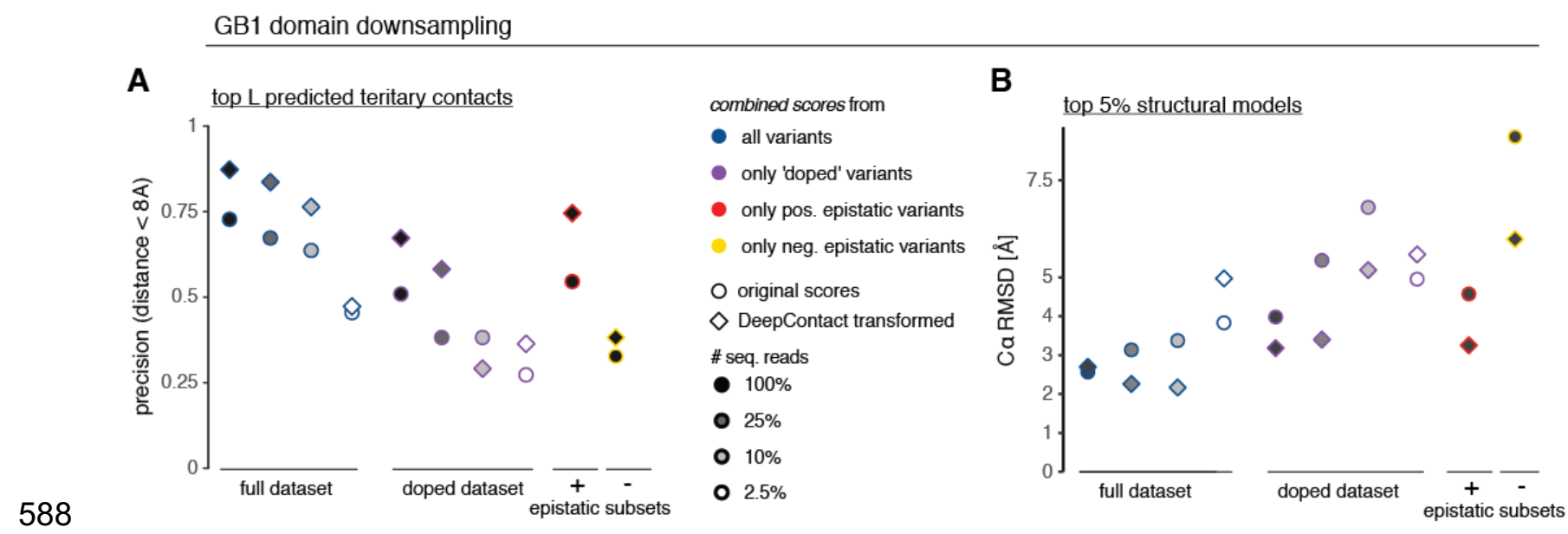
589 Figure 7: Deep learning allows contact and structure prediction from 590 sparser and lower quality datasets

591 A. Precision of top $L$ combined score position pairs for different down-sampled versions of GB1 dataset. Color indicates type of dataset (blue - full dataset, purple - 'doped' dataset, red - only positive epistasis information, yellow - only negative epistasis information), fill indicates number of sequencing reads used in analysis, shape indicates whether DeepContact has been used to transform the interaction score matrix.

B. Accuracy $\langle C \alpha-R M S D\rangle$ of top $5 \%$ structural models derived with tertiary contact restraints from down-sampled GB1 datasets compared to reference structure. Note that for better comparability, for these structural simulations only distance restraints were derived from combined scores but the same secondary structure restraints predicted from PSIPRED and no beta sheet pairing restraints were used for all simulations. Colors and fills as in panel $A$. 


\section{Discussion}

603 We have shown here that simply quantifying the activity of a large number of single and double 604 mutant variants of a macromolecule can provide enough information to determine its high605 resolution 3D structure.

606 We found that although most epistasis within a protein occurs between positions that are not 607 direct structural contacts, aggregation on position pairs, merging of positive and negative 608 epistasis information and partial correlation analysis of epistasis patterns can successfully 609 discriminate direct from indirect structural contacts. Thus, mostly indirect epistatic couplings can 610 be transformed to predict accurate structural contacts and elements. We have shown that this 611 approach works robustly across multiple protein domains and a protein interaction. Moreover, 612 we have demonstrated that the application of a convolutional neural network previously trained 613 on patterns of co-evolution in proteins of known structure both improves structure prediction and 614 allows the use of much lower quality deep mutation datasets.

615 Determining structures by mutagenesis requires an in vitro or in vivo selection assay. For many 616 important molecules and drug targets, specific selection assays based on known functions or 617 interaction partners already exist ${ }^{11,14,15,46,50-55}$. Additionally, many generic selection assays have 618 recently been developed that should allow the stability or functional activity of many proteins to 619 be assayed in vivo without the need for much prior knowledge about the protein under 620 investigation ${ }^{35-38}$. Moreover, many molecules have known interaction partners - proteins, DNA, 621 RNA, or small ligands - for which cis- and trans-epistasis can thus be assessed by binding 622 assays ${ }^{9,55}$. We have shown here how trans-epistasis, for which library design is relatively easy, 623 can lead to information about direct contacts in interaction surfaces as well as in the individual 624 molecules.

625 Although structural information exists in the epistasis maps, our analyses and previous work ${ }^{5-16}$ 626 has shown that many epistatic interactions occur between positions that are not in direct 627 structural contact. Indeed, in the GB1 domain the interactions are strikingly modular, with two 628 mutually exclusive clusters of positive and negative epistatic interactions. This is consistent with 629 many interactions being due to functional or energetic couplings between positions. The cluster 630 of mostly positive epistatic interactions corresponds to a dynamic region involved in lgG binding $631{ }^{11}$. In contrast, the cluster of negative epistatic interactions identifies positions important for the 632 thermodynamic stability of the domain ${ }^{56}$, and the periodicity of local negative epistatic 
633 interactions provide evidence for a shift towards an alternative three-helical conformation that 634 has been previously reported for this sequence family (Extended Data Figure 4E) ${ }^{57}$. This 635 modular organization of epistasis is thus reminiscent of the concept of energetically-coupled 636 protein sectors identified from patterns of sequence co-evolution ${ }^{20,21,58}$.

637 For macromolecules with very large numbers of homologs available in sequence databases, 638 correlated changes in sequence can provide sufficient information for structure determination ${ }^{25-}$ 639 34. However, for many proteins and RNAs insufficient numbers of homologs are available, and 640 for fast evolving, recently-evolved or de novo designed molecules this is a fundamental 641 limitation 26,32,37,39. Moreover, co-evolutionary analysis provides information on the average 642 structure across a large set of homologs, whereas it is easy to envisage how deep mutagenesis 643 could be used to directly determine alternative conformations of macromolecules when they are 644 performing particular selectable functions. The success of evolutionary coupling analysis for 645 predicting the structures of diverse folds and macromolecules does, however, strongly support 646 the generality of the approach outlined here. The demonstration that a deep learning approach 647 previously trained on evolutionary couplings dramatically improves the prediction of contacts 648 from deep mutagenesis data further supports this.

649 As a proof-of-principle we have shown that information from deep mutational scanning 650 experiments alone is sufficient for accurate structure prediction. In practice, however, integration 651 with other structural information is likely to further boost performance. As a first step, we used a 652 deep learning approach, DeepContact, that was trained on evolutionary couplings to learn 653 stereotypical structural patterns in contact maps ${ }^{47}$. DeepContact improved DMS-derived 654 contact prediction precision by up to $30 \%$ for individual proteins (GB1, WW and RRM domains). 655 Moreover, even though it had only been trained on data from individual proteins, it also 656 improved DMS-derived contact predictions for the FOS-JUN protein-protein interaction. 657 Integration with other structural predictors ${ }^{47,59,60}$ and homology-driven structure modeling ${ }^{61,62}$ is 658 likely to further improve accuracy and lower the data quality requirements for structure 659 determination by deep mutagenesis.

660 An analysis of incomplete and down-sampled variants of the GB1 dataset suggests that a high 661 signal-to-noise ratio (measurement range relative to experimental error of fitness estimates), 662 which allows both positive and negative epistasis to be quantified, is an important factor for 663 generating datasets with a quality sufficient for protein structure prediction. For datasets with 664 complete epistasis information, however, sequencing coverage hardly affected prediction 
665 performance. In contrast, prediction performance on the incomplete, 'doped' dataset was

666 sensitive to sequencing coverage. Down to $25 \%$ sequencing coverage, however, performance

667 could be recovered by deep learning. Together, these analyses suggest that our approach

668 should be easily applicable to longer molecules. For example, with the experimental effort

669 undertaken to create and sequence the 55 amino acid GB1 library, a protein of length $\sim 350$

670 amino acids should be assayable at similar prediction performance (using a doped library with

$67125 \%$ sequencing coverage, i.e. $2.5 \%$ of the data: $55 a a / \sqrt{0.025} \approx 350 a a$ ). Such libraries for

672 longer proteins could be created via fragment-based ligation approaches ${ }^{52}$ or via random

673 mutagenesis and barcode-variant linking ${ }^{35}$.

674 Taken together, the results presented here establish deep mutagenesis as a new experimental 675 strategy for structure determination. The approach that we have outlined is not the only one

676 that can be envisaged to predict direct structural contacts from deep mutagenesis data, and 677 other related approaches are also likely to work ${ }^{63}$. The determination of macromolecular 678 structures by physical techniques requires access to very expensive scientific infrastructure. In 679 contrast, deep mutagenesis only requires techniques familiar to many molecular biologists and 680 access to sequencing that is increasingly low cost and available to all. Most importantly, 681 however, deep mutagenesis allows the structures of macromolecules to be studied whilst they 682 are performing particular functions in vitro as well as in vivo in the cell. As such, deep 683 mutagenesis opens up the possibility of low cost and high throughput determination of in vivo 684 macromolecular structures by many molecular biology and genomics labs. A large-scale project 685 to systematically determine the structures of proteins and protein domains should therefore be 686 possible using the existing infrastructure of genomics institutes. 
Table 1: Dataset properties

\begin{tabular}{|l|l|l|l|l|l|l|l|}
\hline Dataset & $\begin{array}{l}\text { Mutated } \\
\text { positions }\end{array}$ & $\begin{array}{l}\text { \% double } \\
\text { mutants }\end{array}$ & $\begin{array}{l}\text { \% doubles } \\
\text { quantifiable\# }\end{array}$ & $\begin{array}{l}\text { \# input reads } \\
\text { positive } \\
\text { epistasis } \\
\text { mutant } \\
\text { (median) }\end{array}$ & $\begin{array}{l}\text { negative } \\
\text { epistasis }\end{array}$ & $\begin{array}{l}\text { measurement } \\
\text { range } \\
\text { (log fitness } \\
\text { units) }\end{array}$ & $\begin{array}{l}\text { relative } \\
\text { error } \\
\text { (median) }\end{array}$ \\
\hline $\begin{array}{l}\text { Protein G } \\
\text { B1 } \\
\text { domain }\end{array}$ & 55 & 97 & 80 & 55 & 248 & 5.8 & $2.8 \%$ \\
\hline $\begin{array}{l}\text { hYAP } \\
\text { WW } \\
\text { domain }\end{array}$ & 33 & 10 & 8.3 & 0.8 & 73 & 0.8 & $8.6 \%$ \\
\hline $\begin{array}{l}\text { Pab1 } \\
\text { RRM2 } \\
\text { domain }\end{array}$ & 25 & 11 & 8.3 & 3.9 & 209 & 3.1 & $3.7 \%$ \\
\hline FOS-JUN & $2 \times 32$ & 43 & 37 & 31 & 124 & 8.6 & $3.6 \%$ \\
\hline
\end{tabular}

689

690

691

692

693

694

695

696

697

698

699

700

701

702

703

704

705

706

707

708

${ }^{\$}$ median percentage of all possible double mutants (361 per position pair) that passed read quality thresholds per position pair

\# median percentage of all possible double mutants (361 per position pair) that passed read quality thresholds and are deemed suitable for epistasis quantification per position pair

* summed number of reads across all input replicates for double mutants that passed read quality thresholds

${ }^{+}$measurement range of selection assay: log fitness range between peak of lethal mutants and the wild-type variant

\& median error of fitness estimates of double mutant variants relative to measurement range of selection assay

\section{Acknowledgements}

We are grateful to Yang Liu and Jian Peng for making their DeepContact code available and for their advice. We thank members of the Lehner lab, T. Gross, G. Mönke, M. Bolognesi and C. Camilloni for discussions and feedback. This work was supported by a European Research Council (ERC) Consolidator grant (616434), the Spanish Ministry of Economy and Competitiveness (BFU2011-26206 and SEV-2012-0208), the AXA Research Fund, the Bettencourt Schueller Foundation, Agencia de Gestio d'Ajuts Universitaris i de Recerca (AGAUR, SGR-831), the EMBL-CRG Systems Biology Program, and the CERCA Program/Generalitat de Catalunya. J.M.S. was supported by an EMBO Long-Term Fellowship (ALTF 857-2016). This project has received funding from the European Union's Horizon 2020 research and innovation programme under the Marie Skłodowska-Curie grant agreement No 752809 (J.M.S). 


\section{Author Contributions}

710 Conceptualization, B.L. and J.M.S.; Methodology, J.M.S.; Investigation, J.M.S.; Writing, J.M.S.

711 and B.L.; Supervision, B.L.

712

713 Competing Interests

714 The authors declare no competing interests.

715

716 Additional Information

717 Correspondence and requests for materials should be addressed to B.L.

\section{References}

7201 Tokuriki, N. \& Tawfik, D. S. Stability effects of mutations and protein evolvability. Current $721 \quad$ Opinion in Structural Biology 19, 596-604, doi:10.1016/j.sbi.2009.08.003 (2009).

7222 Lehner, B. Molecular mechanisms of epistasis within and between genes. Trends in genetics : TIG 27, 323-331, doi:10.1016/j.tig.2011.05.007 (2011).

3 Fowler, D. M. \& Fields, S. Deep mutational scanning: a new style of protein science. Nature Methods 11, 801-807, doi:10.1038/nmeth.3027 (2014).

4 Starr, T. N. \& Thornton, J. W. Epistasis in protein evolution. Protein science : a publication of the Protein Society 25, 1204-1218, doi:10.1002/pro.2897 (2016).

5 Horovitz, A. \& Fersht, A. R. Strategy for analysing the co-operativity of intramolecular interactions in peptides and proteins. $J$ Mol Biol 214, 613-617, doi:10.1016/00222836(90)90275-Q (1990).

6 Carter, P. J., Winter, G., Wilkinson, A. J. \& Fersht, A. R. The use of double mutants to detect structural changes in the active site of the tyrosyl-tRNA synthetase (Bacillus stearothermophilus). Cell 38, 835-840 (1984).

7 Ackermann, E. J., Ang, E. T., Kanter, J. R., Tsigelny, I. \& Taylor, P. Identification of pairwise interactions in the alpha-neurotoxin-nicotinic acetylcholine receptor complex through double mutant cycles. J Biol Chem 273, 10958-10964 (1998).

8 Chen, J. \& Stites, W. E. Energetics of side chain packing in staphylococcal nuclease assessed by systematic double mutant cycles. Biochemistry 40, 14004-14011 (2001). 
7399 Diss, G. \& Lehner, B. The genetic landscape of a physical interaction. eLife 7, 594, 740 doi:10.7554/eLife.32472 (2018).

74110 Melamed, D., Young, D. L., Gamble, C. E., Miller, C. R. \& Fields, S. Deep mutational scanning of an RRM domain of the Saccharomyces cerevisiae poly(A)-binding protein. RNA 19, 1537-1551, doi:10.1261/rna.040709.113 (2013).

11 Olson, C. A., Wu, N. C. \& Sun, R. A comprehensive biophysical description of pairwise epistasis throughout an entire protein domain. Current biology : CB 24, 2643-2651, doi:10.1016/j.cub.2014.09.072 (2014). proximity information and protein model discrimination using saturation-suppressor mutagenesis. eLife 4, 371, doi:10.7554/eLife.09532 (2015). \&amp; Evolution 15, 1, doi:10.1038/s41559-018-0549-8 (2018). Science 352, 837-840, doi:10.1126/science.aae0568 (2016).

Domingo, J., Diss, G. \& Lehner, B. Pairwise and higher-order genetic interactions during the evolution of a tRNA. Nature 558, 117-121, doi:10.1038/s41586-018-0170-7 (2018).

Puchta, O. et al. Network of epistatic interactions within a yeast snoRNA. Science $\mathbf{3 5 2}$, 840-844, doi:10.1126/science.aaf0965 (2016).

Göbel, U., Sander, C., Schneider, R. \& Valencia, A. Correlated mutations and residue contacts in proteins. Proteins 18, 309-317, doi:10.1002/prot.340180402 (1994).

Altschuh, D., Lesk, A. M., Bloomer, A. C. \& Klug, A. Correlation of co-ordinated amino acid substitutions with function in viruses related to tobacco mosaic virus. Journal of molecular biology 193, 693-707 (1987). multiple sequence alignments reveals two classes of coevolving positions. Biochemistry 44, 7156-7165, doi:10.1021/bi050293e (2005).

22 Morcos, F. et al. Direct-coupling analysis of residue coevolution captures native contacts across many protein families. Proceedings of the National Academy of Sciences 108, E1293-1301, doi:10.1073/pnas.1111471108 (2011).

Weigt, M., White, R. A., Szurmant, H., Hoch, J. A. \& Hwa, T. Identification of direct residue contacts in protein-protein interaction by message passing. Proceedings of the National Academy of Sciences 106, 67-72, doi:10.1073/pnas.0805923106 (2009).

Burger, L. \& van Nimwegen, E. Disentangling Direct from Indirect Co-Evolution of Residues in Protein Alignments. PLoS Computational Biology 6, e1000633, doi:10.1371/journal.pcbi.1000633 (2010). 
781

782

783

784

785

786

787

788

789

790

791

792

793

794

795

796

797

798

799

800

801

802

803

804

805

806

807

808

809

810

811

812

813

814

815

816

817

818

819

820

821

822

823

26 Tóth-Petróczy, A. et al. Structured States of Disordered Proteins from Genomic Sequences. Cell 167, 158-170.e112, doi:10.1016/j.cell.2016.09.010 (2016).

27 Hopf, T. A. et al. Three-Dimensional Structures of Membrane Proteins from Genomic Sequencing. Cell 149, 1607-1621, doi:10.1016/j.cell.2012.04.012 (2012).

28 Marks, D. S. et al. Protein 3D structure computed from evolutionary sequence variation. PLOS ONE 6, e28766, doi:10.1371/journal.pone.0028766 (2011).

29 Jones, D. T., Buchan, D. W. A., Cozzetto, D. \& Pontil, M. PSICOV: precise structural contact prediction using sparse inverse covariance estimation on large multiple sequence alignments. Bioinformatics 28, 184-190, doi:10.1093/bioinformatics/btr638 (2012).

30 De Leonardis, E. et al. Direct-Coupling Analysis of nucleotide coevolution facilitates RNA secondary and tertiary structure prediction. Nucleic Acids Research 43, 10444-10455, doi:10.1093/nar/gkv932 (2015).

31 Sułkowska, J. I., Morcos, F., Weigt, M., Hwa, T. \& Onuchic, J. N. Genomics-aided structure prediction. Proceedings of the National Academy of Sciences 109, 1034010345, doi:10.1073/pnas.1207864109 (2012).

32 Ovchinnikov, S. et al. Protein structure determination using metagenome sequence data. Science 355, 294-298, doi:10.1126/science.aah4043 (2017).

33 Ovchinnikov, S. et al. Large-scale determination of previously unsolved protein structures using evolutionary information. eLife 4, e09248, doi:10.7554/eLife.09248 (2015).

34 Ovchinnikov, S., Kamisetty, H. \& Baker, D. Robust and accurate prediction of residueresidue interactions across protein interfaces using evolutionary information. eLife 3, e02030, doi:10.7554/eLife.02030 (2014).

35 Matreyek, K. A. et al. Multiplex assessment of protein variant abundance by massively parallel sequencing. Nature Genetics 50, 874-882, doi:10.1038/s41588-018-0122-Z (2018).

36 Weile, J. et al. A framework for exhaustively mapping functional missense variants. Molecular Systems Biology 13, 957, doi:10.15252/msb.20177908 (2017).

37 Rocklin, G. J. et al. Global analysis of protein folding using massively parallel design, synthesis, and testing. Science 357, 168-175, doi:10.1126/science.aan0693 (2017).

38 Kim, I., Miller, C. R., Young, D. L. \& Fields, S. High-throughput analysis of in vivo protein stability. Molecular \&amp; cellular proteomics : MCP 12, 3370-3378, doi:10.1074/mcp.0113.031708 (2013).

39 Marks, D. S., Hopf, T. A. \& Sander, C. Protein structure prediction from sequence variation. Nature Biotechnology 30, 1072-1080, doi:10.1038/nbt.2419 (2012).

40 Costanzo, M. et al. The genetic landscape of a cell. Science 327, 425-431, doi:10.1126/science.1180823 (2010).

41 Stein, R. R., Marks, D. S. \& Sander, C. Inferring Pairwise Interactions from Biological Data Using Maximum-Entropy Probability Models. PLoS Computational Biology 11, e1004182, doi:10.1371/journal.pcbi.1004182 (2015).

42 Andreani, J. \& Söding, J. bbcontacts: prediction of $\beta$-strand pairing from direct coupling patterns. Bioinformatics 31, 1729-1737, doi:10.1093/bioinformatics/btv041 (2015). 
824

825

826

827

828

829

830

831

832

833

834

835

836

837

838

839

840

841

842

843

844

845

846

847

848

849

850

851

852

853

854

855

856

857

858

859

860

861

862

863

864

865

43 Jones, D. T. Protein secondary structure prediction based on position-specific scoring matrices. Journal of molecular biology 292, 195-202, doi:10.1006/jmbi.1999.3091 (1999).

44 The PyMOL Molecular Graphics System, V. S., LLC. The PyMOL Molecular Graphics System, Version 1.8 (2015).

45 Schwieters, C. D., Kuszewski, J. J., Tjandra, N. \& Clore, G. M. The Xplor-NIH NMR molecular structure determination package. Journal of magnetic resonance (San Diego, Calif. : 1997) 160, 65-73 (2003).

46 Araya, C. L. et al. A fundamental protein property, thermodynamic stability, revealed solely from large-scale measurements of protein function. Proceedings of the National Academy of Sciences 109, 16858-16863, doi:10.1073/pnas.1209751109 (2012).

47 Liu, Y., Palmedo, P., Ye, Q., Berger, B. \& Peng, J. Enhancing Evolutionary Couplings with Deep Convolutional Neural Networks. Cell systems, doi:10.1016/j.cels.2017.11.014 (2017).

48 Schaarschmidt, J., Monastyrskyy, B., Kryshtafovych, A. \& Bonvin, A. M. J. J. Assessment of contact predictions in CASP12: Co-evolution and deep learning coming of age. Proteins 86 Suppl 1, 51-66, doi:10.1002/prot.25407 (2018).

49 Fox, N. K., Brenner, S. E. \& Chandonia, J. M. SCOPe: Structural Classification of Proteins--extended, integrating SCOP and ASTRAL data and classification of new structures. Nucleic Acids Res 42, D304-309, doi:10.1093/nar/gkt1240 (2014).

50 Starita, L. M. et al. Massively Parallel Functional Analysis of BRCA1 RING Domain Variants. Genetics 200, 413-422, doi:10.1534/genetics.115.175802 (2015).

51 Starita, L. M. et al. Activity-enhancing mutations in an E3 ubiquitin ligase identified by high-throughput mutagenesis. Proceedings of the National Academy of Sciences 110, E1263-1272, doi:10.1073/pnas.1303309110 (2013).

52 Poelwijk, F. J., Socolich, M. \& Ranganathan, R. Learning the pattern of epistasis linking genotype and phenotype in a protein. bioRxiv, doi:10.1101/213835 (2017).

53 Starr, T. N., Picton, L. K. \& Thornton, J. W. Alternative evolutionary histories in the sequence space of an ancient protein. Nature 5, e16965, doi:10.1038/nature23902 (2017).

54 Fowler, D. M. et al. High-resolution mapping of protein sequence-function relationships. Nature Methods 7, 741-746, doi:10.1038/nmeth.1492 (2010).

55 McLaughlin Jr, R. N., Poelwijk, F. J., Raman, A., Gosal, W. S. \& Ranganathan, R. The spatial architecture of protein function and adaptation. Nature 491, 138-142, doi:10.1038/nature11500 (2012).

56 Otwinowski, J. Biophysical inference of epistasis and the effects of mutations on protein stability and function. Mol Biol Evol, doi:10.1093/molbev/msy141 (2018).

57 Alexander, P. A., He, Y., Chen, Y., Orban, J. \& Bryan, P. N. A minimal sequence code for switching protein structure and function. Proceedings of the National Academy of Sciences 106, 21149-21154, doi:10.1073/pnas.0906408106 (2009).

58 Salinas, V. H. \& Ranganathan, R. Coevolution-based inference of amino acid interactions underlying protein function. eLife 7, 41, doi:10.7554/eLife.34300 (2018). 
866

867

868

869

87060

871

872

873

874

875

876

877

878

879

880

881

882

883

884

885

886

887

888

889

890

891

892

893

894

895

896

897

898

899

900

901

902

903

904

905

906

907

908

59 Jones, D. T., Singh, T., Kosciolek, T. \& Tetchner, S. MetaPSICOV: combining coevolution methods for accurate prediction of contacts and long range hydrogen bonding in proteins. Bioinformatics 31, 999-1006, doi:10.1093/bioinformatics/btu791 (2015).

60 Wang, S., Sun, S., Li, Z., Zhang, R. \& Xu, J. Accurate De Novo Prediction of Protein Contact Map by Ultra-Deep Learning Model. PLoS Computational Biology 13, e1005324, doi:10.1371/journal.pcbi.1005324 (2017).

61 Rohl, C. A., Strauss, C. E., Misura, K. M. \& Baker, D. Protein structure prediction using Rosetta. Methods Enzymol 383, 66-93, doi:10.1016/S0076-6879(04)83004-0 (2004).

62 Yang, J. et al. The I-TASSER Suite: protein structure and function prediction. Nat Methods 12, 7-8, doi:10.1038/nmeth.3213 (2015).

63 Rollins, N. J. et al. 3D protein structure from genetic epistasis experiments. doi:10.1101/320721 (2018).

64 Rubin, A. F. et al. A statistical framework for analyzing deep mutational scanning data. Genome biology 18, 741, doi:10.1186/s13059-017-1272-5 (2017).

65 Edgar, R. C. Search and clustering orders of magnitude faster than BLAST. Bioinformatics 26, 2460-2461, doi:10.1093/bioinformatics/btq461 (2010).

66 Barlow, R. Statistics : a guide to the use of statistical methods in the physical sciences. (Wiley, 1989).

67 Schäfer, J. \& Strimmer, K. A shrinkage approach to large-scale covariance matrix estimation and implications for functional genomics. Statistical applications in genetics and molecular biology 4, Article32, doi:10.2202/1544-6115.1175 (2005).

68 Gallagher, T., Alexander, P., Bryan, P. \& Gilliland, G. L. Two crystal structures of the B1 immunoglobulin-binding domain of streptococcal protein $G$ and comparison with NMR. Biochemistry 33, 4721-4729 (1994).

69 Pires, J. R. et al. Solution structures of the YAP65 WW domain and the variant L30 K in complex with the peptides GTPPPPYTVG, N-(n-octyl)-GPPPY and PLPPY and the application of peptide libraries reveal a minimal binding epitope. Journal of molecular biology 314, 1147-1156, doi:10.1006/jmbi.2000.5199 (2001).

70 Deo, R. C., Bonanno, J. B., Sonenberg, N. \& Burley, S. K. Recognition of polyadenylate RNA by the poly(A)-binding protein. Cell 98, 835-845 (1999).

71 Glover, J. N. \& Harrison, S. C. Crystal structure of the heterodimeric bZIP transcription factor c-Fos-C-Jun bound to DNA. Nature 373, 257-261, doi:10.1038/373257a0 (1995).

72 Adhikari, B., Bhattacharya, D., Cao, R. \& Cheng, J. CONFOLD: Residue-residue contact-guided ab initio protein folding. Proteins 83, 1436-1449, doi:10.1002/prot.24829 (2015).

73 Zhang, Y. \& Skolnick, J. Scoring function for automated assessment of protein structure template quality. Proteins 57, 702-710, doi:10.1002/prot.20264 (2004).

74 Seemayer, S., Gruber, M. \& Söding, J. CCMpred--fast and precise prediction of protein residue-residue contacts from correlated mutations. Bioinformatics 30, 3128-3130, doi:10.1093/bioinformatics/btu500 (2014). 


\section{Methods:}

\section{Datasets and preprocessing}

\section{Protein G B1 domain}

912 Protein G B1 domain (GB1) deep mutational scanning data was obtained from the 913 supplementary information of Olson, et al. ${ }^{11}$. The data consists of summed read counts of three 914 replicate experiments assaying the binding affinity of GB1 variants to immunoglobulin $\mathrm{G}$ (IgG).

915 Read frequencies of each single or double mutant variant in input library and output library (after 916 binding affinity assay) were calculated as variant read counts relative to wild-type variant read counts. A variant's fitness was calculated as the natural logarithm of the ratio of output to input

918 read frequency, i.e. $f_{i}=\log \left(\frac{n_{i}^{\text {out }} / n_{w t}^{\text {out }}}{n_{i}^{\text {in }} / n_{w t}^{\text {in }}}\right)$, with $\mathrm{n}$ as read counts, superscripts denoting input or output sequencing library and subscripts denoting variant $i$ or wild-type variant.

The standard error of fitness estimates was calculated from read counts under Poissonian

921 assumptions, i.e. $\sigma_{i}=\sqrt{\frac{1}{n_{i}^{\text {in }}}+\frac{1}{n_{i}^{\text {out }}}+\frac{1}{n_{w t}^{\text {in }}}+\frac{1}{n_{w t}^{\text {out }}}} 64$. We note that this is a lower bound estimate of 922 the actual error, due to the lack of replicate information.

923 Each measurement assay has a lower measurement limit due to unspecific background effects 924 (Extended Data Figure 2A). In the case of the IgG-binding assay for GB1, this is presumably 925 mainly due to unspecific carry-over on beads ${ }^{11}$. The fitness values derived from the 926 measurement are therefore a convolution of the actual binding affinities to $\lg G$ and nonspecific 927 carryover, i.e. $\exp \left(f_{i}^{\text {measured }}\right)=\exp \left(f_{i}^{\text {binding }}\right)+\exp \left(f^{\text {carryover }}\right)$. Fitness values of variants 928 close to the lower measurement limit of the assay are therefore dominated by unspecific 929 carryover effects. The lower measurement limit of the assay was estimated by two approaches 930 that yielded identical estimates. One, from a kernel density estimate of the single mutant fitness 931 distribution ( $\mathrm{R}$ function density with parameter bw set to 0.15 ), where the position of the lower 932 mode of the data corresponded to $f^{\text {carryover }}=-5.85(\sim 0.3 \%$ on linear scale). Two, from 933 examining the fitness distribution of double mutants with expected fitness lower than -8 log934 units, i.e. double mutants resulting from two lethal or nearly lethal single mutant variants, whose 935 fitness values are thus expected to be dominated by background effects. The median of this 936 background fitness distribution yielded an estimate of $f^{\text {carryover }}=-5.85$. 
$9377 \%$ of double mutant variants were discarded due to too low sequencing coverage in input or

938 output libraries (Extended Data Figure 2B). That is, variants that had less than 200 input reads

939 and no output reads were discarded, because it is not possible to determine their fitness. Above

940200 input reads, variants without output reads are certain to be dominated by nonspecific

941 carryover effects. These variants were retained and their fitness was calculated by setting their

942 output read count to 0.5 .

\section{GB1 down-sampling}

944 Down-sampling of the full GB1 dataset was performed in three different ways.

945 For the 'doped' datasets, we only allowed amino acid changes created by one nucleotide 946 mutation from the wild-type sequence (ENA entry M12825). To down-sample the sequencing 947 read coverage, for each variant we picked as a down-sampled read count the draw of 948 successes from a binomial distribution with the number of sequencing reads in the full datasets 949 as trials and the target down-sampling rate $(25 \%, 10 \%$ or $2.5 \%)$ as chance of success. For the 950 read down-sampled and doped datasets (and combinations of both), the analysis workflow for 951 the full dataset was repeated.

952 For the down-sampled datasets taking only positive or negative epistatic information into 953 account, we calculated epistasis and association scores from epistatic enrichment matrices and 954 partial correlation matrices of only positive or negative epistasis information. Instead of merging 955 positive and negative matrices and then calculating z-scores, we only calculated z-scores with 956 the individual errors from positive or negative epistasis information. The combined scores (for 957 which results are reported) for each set was then calculated as for the full dataset by summing 958 standardized epistasis and association scores.

\section{9 hYAP WW domain}

960 hYAP WW domain data was obtained from Sequence Read Archive (SRA) entry SRP015751 46. 961 Paired-end reads were merged with USearch ${ }^{65}$ and merged reads with any base having a 962 Phred base quality score below 20 were discarded. Read counts from the two technical 963 sequencing replicates were merged and read counts for the same amino acid variants with at 964 most one synonymous mutation in another codon were summed up. The dataset consists of an 965 input library and three output libraries after consecutive rounds of selection in a phage display 966 assay. Fitness was estimated as the slope of log frequency (variant counts divided by wild-type 967 counts) changes over the rounds of selection experiment ${ }^{46}$. For each variant at each selection 
968 step a Poissonian error of $\sigma_{i, x}=\sqrt{\frac{1}{n_{i}^{x}}+\frac{1}{n_{w t}^{x}}}$ was calculated, with $\mathrm{x}$ denoting the selection step.

969 Slopes were calculated as weighted straight line least square fits ${ }^{66}$. Comparison of library-wide 970 changes in variant frequencies between selection rounds suggested differential selection 971 pressures across the rounds. We thus applied a non-equidistant spacing of $0.6,1.17$ and 1.22 972 between selection rounds when calculating slopes. Only variants that have more than 10 reads 973 in the input library and at least one read after the first selection were retained for further analysis 974 (45\% of constructed double mutants). The lower fitness limit was calculated as the weighted 975 mean fitness of all variants containing STOP codons (-0.78 in log-fitness units).

976 Pab1 RRM2 domain

977 Pab1 RRM2 domain data was obtained from the Supplementary Table 5 of Melamed, et al. ${ }^{10}$. 978 Reported enrichment scores were log-transformed to obtain fitness values. Output reads per 979 variant were deduced from the number of input reads times the enrichment score and used to 980 calculate a Poissonian error of the fitness estimate. Single mutant count data is not provided 981 and we thus estimated the error of single mutant fitness estimates to be 0.01 . Lower bound of 982 fitness assay was estimated as weighted mean fitness of all double mutant variants containing 983 STOP codons (-3.1 log-fitness units).

\section{FOS-JUN interaction}

985 Raw count tables were provided by Guillaume Diss ${ }^{9}$. The dataset consists of input and output sequencing libraries after selection for physical interaction between the two proteins in a protein complementation assay in three biological replicates. Per sequencing library, read counts from all synonymous variants were summed up. Only variants that had more than 10 reads in each of the three input libraries were used for further analysis ( $43 \%$ of double mutants). Per input/output replicate, fitness of each variant was calculated as the log change in frequency compared to the wild-type variant (as for GB1). A Poissonian error for each variant's fitness estimate was 992 derived.

993 The dataset has a large dynamic range, thus many low-fitness variants with low input read 994 coverage have very low or no output read counts (per replicate $\sim 1 / 3$ of variants have below 3 995 output counts, $\sim 15 \%$ of variants have zero output counts), effectively reducing the dynamic 996 range of the assay for low input variants and distorting the estimate of the overall fitness 997 distribution (see Extended Data Figure 5E). To overcome this, we implemented a Bayesian 998 estimator of fitness. For each double mutant variant, we first identified the 1000 nearest 
neighbors in single mutant fitness space (i.e. those double mutants whose respective single mutant fitness values are similar to the single mutant fitness values of the variant under consideration) with sufficient input coverage (more than 100 reads in the input library). From this set of 1000 nearest neighbors we calculated the expected distribution of double mutant fitness values, which served as a prior distribution. For the variant under consideration we calculated the likelihood distribution of fitness values given its input and output read counts under Poissonian assumptions. Fitness was then estimated as the mean of the distribution resulting from the multiplication of prior and likelihood distributions. Error of fitness estimate was estimated as the standard deviation of the resulting distribution. Estimated fitness from the three replicate experiments were merged by weighted averaging.

\section{Epistasis classification}

1011 Epistasis was calculated from a non-parametric null model (Figure 1B) in order to account for 1012 nonlinearities close to the lower limit of the fitness assay measurement range, non-specific 1013 epistatic behavior resulting from e.g. thermodynamic stability thresholds as well as differential 1014 uncertainty of fitness measurements across the fitness landscape, due to lower read counts in 1015 the output for low fitness variants.

1016 First, double mutant fitness values were corrected by subtracting the average local fitness computed using a two-dimensional local polynomial regression (using $\mathrm{R}$ function loess with span $=0.2$ ). This was necessary to avoid boundary effects of quantile-based fits in boundary regions with non-zero slopes. 5th and 95th percentile surfaces were then fit to these corrected double mutant fitness values, by computing for each double mutant variant the 5th and 95th percentile of the fitness distribution made up of the $1 \%$ closest neighbors in single mutant 1022 fitness space. Double mutant variants with fitness values below the 5th or above the 95th 1023 percentile were categorized as negative or positive epistatic, respectively (Figure 1B).

1024 The evaluation of positive or negative epistasis was, however, restricted to specific subsets of 1025 the data where measurement errors do not impede epistasis classification (Extended Data 1026 Figure 2C). The data subset deemed suitable for positive epistasis classification is limited to 1027 regions where

1028 the 95th percentile fitness surface is below wild-type fitness 
- at least one single mutant fitness value is significantly smaller than wild-type fitness at two standard errors

- the expected fitness (sum of both single mutant fitness values) is not significantly higher than wild-type at two standard errors

The rationale for these criteria is to avoid double mutants from two neutral single mutants, because these are dominated by measurement noise of overabundant wild-type like variants. No restrictions were instead applied to the lower limits of the measurement range, because otherwise no/little epistasis quantification would have been available for several positions with very strong detrimental effects as well as because strong positive epistatic effects are observed in these regions, despite the dominance of background measurement effects.

The data subset in which variants were potentially classified as negative epistatic is limited to data regions where

- the 5th percentile fitness surface is above the 95th percentile of the background effect distribution; this value is derived from the 95th percentile of double mutant fitness values with expected fitness below -8 (analogous to lower fitness limit estimation, see above).

- both single mutant fitness values are significantly higher than the lower limit of the fitness assay measurement range at two standard errors

- the expected fitness (sum of both single mutant fitness values) is not significantly higher than wild-type at two standard errors

The rationale for criteria 1 and 2 is to avoid background measurement effects that make negative epistasis quantification unreliable.

As a result of these restrictions as well as differences in initial coverage, the number of double mutant variants that can be used to assess positive and negative epistasis varies substantially across position pairs and datasets (see Table 1, Extended Data Figures 2D-F and 5A\&D).

\section{Epistatic interactions (epistasis scores)}

We derived several interaction scores to estimate which position pairs are in close contact in the tertiary structure (see Figures 2A and 3A,B and Extended Data Figure 1). These scores are based on summarizing epistasis information on the position pair-level and accounting for the 
1058

1059

1060

1061

1062

1063

1064

1065

1066

1067

1068

1069

1070

1071

1072

1073

1074

1075

1076

1077

1078

1079

1080

1081

1082

1083

1084

1085

1086

1087

1088

1089

uncertainty inherent in the summarized estimates due to differential error of fitness estimates across the measurement range as well as varying numbers of double mutants amenable to epistasis classification (see Table 1, Extended Data Figures 2D-F and 5A\&D).

To summarize epistasis information on the position pair-level, we calculated the fraction of positive or negative epistatic variants per position pair. The fraction of positive epistatic variants per position pair is the number of positive epistatic variants divided by the number of all variants that lie in the double mutant space amenable to positive epistasis classification (Extended Data Figure 1, step 5b, equivalent calculation for negative epistasis fraction). Because enrichments with positive and negative epistatic variants per position are anti-correlated (Extended Data Figure $3 A$ ), we treated both separately and only aggregated them to derive the final interaction scores.

To estimate the uncertainty in epistatic fractions per position pair for downstream analyses we implemented a re-sampling approach (Extended Data Figure 1, step 5, described here for positive epistatic variants, but equivalent for negative epistatic variants). In each of 10.000 resampling runs:

- each variant's fitness was drawn from a normal distribution with the measured fitness as mean and the uncertainty due to sequencing coverage as standard deviation $f_{i j}^{\text {sampled }}=$ $\mathcal{N}\left(f_{i j}, \sqrt{\sigma_{i j}^{2}+s_{i}^{2} * \sigma_{i}^{2}+s_{j}^{2} * \sigma_{j}^{2}}\right)$, with $s_{x}$ as the local slope of the median fitness landscape in direction of the respective single mutant (step 5a)

- positive epistasis of variants was re-classified given the drawn fitness values (also step 5a)

- each position pair's fraction of positive epistatic variants was drawn from the posterior probability distribution of how likely an underlying true fraction of epistatic variants $E_{x y}^{+}$is to generate the observed fraction of epistatic variants given the finite number of overall variants, i.e. $\mathrm{e}_{x y}^{+} \sim \mathrm{p}\left(\mathrm{E}_{x y}^{+} \mid \# \varepsilon_{x y}^{+}\right.$, \# variants $x y$ ) (step $\left.5 \mathrm{~b}\right)$. The posterior probability distribution is the product of a prior probability distribution - the kernel density estimate of the expected epistatic fractions across all position pairs (calculated using $\mathrm{R}$ function density with parameter bw set to 0.05) - and the likelihood function for the underlying true fraction of epistatic variants given the observed fraction of epistatic variants and the overall number of variants under binomial sampling assumptions

To derive an interaction score from the epistatic fractions per position pair, mean positive and negative epistatic fractions across resampling runs were combined by weighted averaging, with 
1090

1091

1092

1093

1094

weights as the inverse variances of epistatic fractions across resampling runs, i.e. $\left\langle e_{x y}\right\rangle=$ $\frac{\left\langle e_{x y}^{+}\right\rangle * \sigma_{e_{x y}^{+}}^{-2}+\left\langle e_{x y}^{-}\right\rangle * \sigma_{e_{x y}^{-}}{ }^{-2}}{\sigma_{e_{x y}^{+}}{ }^{-2}+\sigma_{e_{x y}^{-}}^{-2}}$.

To arrive at the final epistasis score, the mean epistatic fractions were further normalized by their combined uncertainty, $E_{x y}=\left\langle e_{x y}\right\rangle / \sigma_{x y}$, with $\sigma_{x y}=\left({\sigma_{e_{x y}^{+}}}^{-2}+{\sigma_{e_{x y}^{-}}}^{-2}\right)^{-1 / 2}$ (step 6).

\section{Epistasis pattern correlations (association scores)}

In addition to the epistasis score we derived an interaction score from the partial correlation of epistasis patterns between position pairs, termed association score. The rationale behind this score is that proximal positions in the protein should have similar distances and geometrical arrangements towards all other positions in the protein and should therefore also have similar patterns of epistatic interactions with all other positions.

In each re-sampling run we constructed a symmetric matrix of the drawn positive epistatic fractions $e_{x y}^{+}$with number of rows and columns as the number of mutated positions (Extended Data Figure 1, step 5c, equivalent for negative epistatic fractions). Missing values (positions pairs without observed variants) were imputed by drawing a random value from the overall distribution of epistatic fractions. A pseudo count equal to the first quartile of the epistatic fraction distribution was added to each epistatic fraction. Diagonal elements (epistatic fractions of a position with itself) were set to 1 . The matrix values were transformed by the natural logarithm (to make distributions more symmetric, thus correlations are not dominated by few position pairs with large epistatic fractions) and for each pair of columns the Pearson correlation coefficient was calculated to arrive at the correlation matrix (step 5d).

A shrinkage approach was used to improve the estimate of the correlation matrix ${ }^{67}$. In brief, the empirical correlation matrix is shrunk towards the identity matrix in order to minimize the meansquared error between estimated and true correlation matrix. Additionally, this yields a positive definite and well-conditioned correlation matrix, suitable for inversion. All computations on correlation matrices, shrinkage and matrix inversion were performed with the $R$ package corpcor, functions cor.shrink and pcor.shrink ${ }^{67}$.

Partial correlations of epistatic patterns between each position pair were calculated by inverting the shrunk correlation matrix and normalizing each off-diagonal entry of the inverted matrix by 
1119 the geometric mean of the two respective diagonal entries, i.e. $a_{x y}^{+}=\frac{r_{x y}^{-1}}{\sqrt{r_{x x}^{-1} * r_{y y}^{-1}}}$, with $r_{x y}^{-1}$ as the $1120(\mathrm{x}, \mathrm{y})$-entry of the inverted correlation matrix (Extended Data Figure 1, step 5d). Equivalent to the 1121 epistasis score, positive and negative partial correlation estimates were merged by calculating 1122 weighted averages of their mean estimates across re-sampling runs, with weights as the inverse 1123 variances across resampling runs, i.e. $\left\langle a_{x y}\right\rangle=\frac{\left\langle a_{x y}^{+}\right\rangle * \sigma_{a_{x y}^{+}}{ }^{-2}+\left\langle a_{x y}^{-}\right\rangle * \sigma_{a_{x y}^{-}}{ }^{-2}}{\sigma_{a_{x y}^{+}}{ }^{-2}+\sigma_{a_{\bar{x} y}^{-}}{ }^{-2}}$, and the final association 1124 score normalized by the combined uncertainty, $A_{x y}=\left\langle a_{x y}\right\rangle / \sigma_{x y}$, with $\sigma_{x y}=\left(\sigma_{a_{x y}^{+}}{ }^{-2}+\right.$ $\left.1125 \sigma_{a_{x y}^{-}}{ }^{-2}\right)^{-1 / 2}($ step 6).

\section{Aggregating epistasis and association scores (combined scores)}

1128 We further derived a combined score by summing the standardized epistasis and association scores, i.e. $C_{x y}=\frac{E_{x y}-\langle E\rangle}{\sigma_{E}}+\frac{A_{x y}-\langle A\rangle}{\sigma_{A}}$. We note that this is a naïve approach to combining the information from these two complementary sources, and surely more sophisticated approaches that further improve proximity estimates can be developed.

\section{Secondary structure prediction}

1134 We used a two-dimensional kernel smoothing approach to predict secondary structure elements 1135 from interaction score matrices (Figure 4A-C). For a given position along the linear chain (on the 1136 diagonal of the interaction score matrix), interaction scores (off the diagonal) are integrated with 1137 distance-specific weighting according to the kernel, which reflects the known geometry of 1138 secondary structures.

1139 The alpha kernel takes on a sinusoidal profile perpendicular to the diagonal to weight 1140 interactions according to whether the position pair considered should have congruent side-chain 1141 orientations (see diagonal and perpendicular profiles in Figure 4B). The kernel was defined as $1142 k_{\alpha}(d, p)=\left(\cos \left(p * \frac{2 \pi}{3.6}\right)+1 / 3\right) * e^{-\frac{d^{2}}{c^{2}}}$, with $d=|2 x-i-j|$ as the diagonal distance of the 1143 interaction $i j$ (off the diagonal) to the reference position $x$ (on the diagonal) and $p=|i-j|$ as the 1144 perpendicular distance of the interaction off the diagonal. The kernel weight for positions with $p$ 
$1145>5$ was set to 0 , thus only including interactions across little more than the first helical turn.

1146 Finally, $c=4$ is the integration scale for the Gaussian kernel along the diagonal. While smaller

1147 integration scales do yield nosier results and longer integration scales can lead to non-detection

1148 of shorter secondary structure stretches, we found that in practice our whole approach

1149 (including the actual detection algorithm described below) is robust to alterations of the 1150 integration length.

1151 The kernel smoothed alpha value for a given position $\mathrm{x}$ on the diagonal is then calculated as the 1152 sum over all interaction scores times their kernel weights $K_{\alpha, x}=\sum_{i} \sum_{j} k_{\alpha}(d, p) * S_{i j}$, where $S_{i j}$ is 1153 one of the interaction scores (epistasis, association or combined score) at position pair ij.

1154 The beta kernel takes an alternating profile perpendicular to the diagonal to weight interactions 1155 according to alternating side-chain orientations in a beta strand and was defined as $k_{\beta}(d, p)=$ $1156((p+1) \bmod 2-1 / 3) * e^{-\frac{d^{2}}{c^{2}}}$, with $c=4$. Only interactions with perpendicular distances equal 1157 or smaller than two (i.e. $k_{\beta}(d, p>3)=0$ ) were included.

1158 To calculate whether kernel-weighted interaction scores of a specific position are larger than 1159 expected, they were compared to kernel-weighted scores obtained from $10^{4}$ randomized control 1160 datasets. Randomization was performed by shuffling all interaction scores, while preserving 1161 matrix symmetry, and kernel-weighted interaction scores from randomized control datasets 1162 were calculated for each position independently to control for possible boundary effects in 1163 positions close to the borders of the protein chain. A p-value for each position was calculated as 1164 the fraction of random controls with kernel smoothed values above that of the real data.

1165 Secondary structure elements were identified by searching for continuous stretches of positions 1166 with high propensities to belong to either alpha helices or beta strands. The following workflow 1167 was implemented:

1168 1. calculate a combined $p$-value for seeds of length 3 by combining position-wise $p$-values using Fisher's method for both alpha and beta kernel smoothed interaction scores

2. separate positions according to whether combined p-values of seeds from alpha or beta kernels are more significant, i.e.

1172 2.1. for alpha helical propensity calculations only consider stretches of at least 5 consecutive positions for which the combined $p$-value of seeds for alpha kernel smoothing is smaller than that from beta kernel smoothing (thus setting the lower size limit of alpha helical elements to five) 
2.2. for beta strand propensity calculations only consider stretches of at least 3 consecutive positions for which the combined p-value of seeds for beta kernel smoothing is smaller than that from alpha kernel smoothing (thus setting the lower size limit of beta strands to three)

For alpha helices and beta strands separately and while combined p-values of seeds $<0.05$

3. select the most significant seed

4. test whether extension to any side yields lower combined p-value 4.1. if yes: extend seed in this direction and repeat step 4

4.2. else: repeat step 4 once to see whether further extension in same direction yields lower combined p-value

4.2.1. if yes: extend and repeat step 4

4.2.2. else: proceed to step 5

5. fix as secondary structure element and delete all 'used' $p$-values (and combined seed $p$ values), such that other elements cannot incorporate them

6. check whether other already fixed elements are adjacent or at most one position away

6.1. if yes: merge both elements

7. repeat steps 3-6 until no more seeds with combined $p$-value $<0.05$ are left

This yields a list of predicted locations of secondary structure elements. We note that the secondary structure elements predicted from deep mutational scanning data could be compared to and combined with predictions derived from other tools, such as PSIPRED (Jones, 1999), to further improve reliability.

To detect beta sheet interactions a modified beta strand kernel was used. In contrast to beta strand detection, the beta sheet interaction kernel is centered on each off-diagonal position. For beta sheet kernels diagonal and perpendicular distances are therefore modified as $d=$ $|x+y-i-j|$ and $p=|x-i-(y-j)|$. The kernels to detect parallel and anti-parallel beta sheets differ in which is their 'diagonal' direction, i.e. the direction at which consecutive position pairs interact in the beta sheet (Extended Data Figure 4A). Therefore, parameters $d$ and $p$ were swapped for the anti-parallel beta sheet kernel. Also, because these positions can be deemed the most crucial for deciding whether a position participates in a beta sheet interaction or not, we up-weighted these positions (those with $p=0$ ) in the kernel by a factor of two, i.e. $K_{\beta}(d, 0)=$ $4 / 3 * e^{-\frac{d^{2}}{c^{2}}}$ 
Beta sheet interactions were identified by searching for the most significant stretches of parallel and anti-parallel interactions (similar to workflow for alpha helices and beta strands), then identifying the set of most significant interactions that is consistent with previously predicted secondary structure elements.

1211 In particular, step 1 \& 3-7 from the above-described workflow were performed for the parallel 1212 beta sheet kernel on each sub-diagonal (parallel to the main diagonal) of the interaction score 1213 matrix separately; and for the anti-parallel beta sheet kernel on each perpendicular diagonal of 1214 the interaction score matrix separately.

1215 The steps were modified as follows:

- for anti-parallel beta sheet interactions, only positions with a distance greater than 1 from the main diagonal were used to calculate seed p-values (assuming anti-parallel beta

We extended the workflow with the following steps to predict beta sheet interactions within the protein domain:

8. compute association of seeds with known beta strands (e.g. seed positions overlap strand 1 on one side and coincide with strand 3 on the other side)

9. while there are seeds with $p<0.05$ : pick most significant seed from either the parallel or anti-parallel sheet subset

10. check consistency with secondary structure elements

10.1. discard the seed and jump back to step 9 if

10.1.1. it is overlapping or too close to an alpha helix or the linker region between two beta strands that interact (minimal distance smaller one)

10.1.2. at least one of the two strands it is associated with already has two other beta sheet interactions or the total number of beta sheet interactions exceeds $2^{*}$ (\#beta strands - 1)

\section{2. modify secondary structure elements and start anew from step 3 if}

10.2.1. one side of the seed is not associated to a known beta strand: create this beta strand

10.2.2. if both sides of the seed are associated with the same known strand: split the strand and create a linker region in-between the strands 
10.3. else fix the beta sheet interaction and delete all other interactions that are associated with the same strands and haven't been fixed yet, jump back to step 9

1242 11. if no more seeds with $p<0.001$, finish

1243 12. update beta strands: keep only those positions that are part of a beta sheet interaction

1245 For beta sheet pairing detection in the GB1 domain (as reported in Figure 4D and Extended 1246 Data Figures 4A-C and 7B) we used as input the secondary structure element predictions 1247 derived from the deep mutational scanning data (as shown in Figure 4A-C and Extended Data 1248 Figure 4D). For the RRM and WW domains, we used as input PSIPRED predicted secondary 1249 structure elements, due to the insufficient signal from secondary structure element predictions 1250 from deep mutational scanning data.

\section{Protein distance metrics}

1253 The minimal side chain heavy atom distance, i.e. the minimal distance between any two side1254 chain heavy atoms of a position pair (in case of glycine, $\mathrm{C} \alpha$ ), was used as the general distance 1255 measure. A direct contact was defined as minimal side-chain heavy atom distance $<8 \AA$. For all 1256 evaluations of predicted contact precision we only considered position pairs with non-trivial 1257 tertiary contacts (those with a linear sequence separation of greater than 5 positions).

1258 We do find that, while using all heavy atoms to calculate distances increase the true positive 1259 rates of predicted contacts by about $10 \%$, side-chain heavy atom distances display much higher 1260 true positive rates over random expectation, thus suggesting that side-chain interactions are 1261 more informative for epistatic interactions (Extended Data Figure 7C).

1262 The Floyd-Warshall algorithm (implemented as custom script in R) was used to calculate the 1263 minimal number of edges $<8 \AA$ that connect any two positions in the protein.

1264 Reference structures used as comparison were

1265 - GB1 domain: PDB entry 1pga, X-ray diffraction structure ${ }^{68}$

1266 - WW domain: PDB entry $1 \mathrm{kgq}$, solution NMR structure ${ }^{69}$

1267 - RRM domain: PDB entry 1cvj (chain A), X-ray diffraction structure of human Pab1 ${ }^{70}$; 1268 note that the central section of the yeast RRM domain analysed is one nucleotide longer 1269 than the corresponding homologous region in the human RRM domain. We thus 
arbitrarily removed position 14 (in the loop region) when comparing the DMS-derived predictions to the human Pab1 structure.

We found that precision or accuracy calculated against other reference structures differed only marginally, thus we have limited reporting to the aforementioned PDB entries.

\section{Protein folding}

1277 To ab initio determine protein structures, we performed simulated annealing using the XPLOR$1278 \mathrm{NIH}$ modeling suite ${ }^{45}$ with structural restraints derived from the deep mutational scanning data. 1279 Simulations were performed in three stages, in each of which 500 structural models were 1280 generated. Stages 1 and 2 served to identify inconsistencies among defined structural restraints. Additionally, in stage 2 an average structure of the best $10 \%$ of models was calculated. Stage 3 served to refine this average structure to obtain a final set of best models.

1283 Restraints from top predicted contacts (position pairs with highest interaction scores and linear chain separation greater than 5 positions) were implemented by setting $C \beta-C \beta$ atom distances ( $\mathrm{C} \alpha$ in case of Glycine) between positions to range between 0 and $8 \AA$ and weighting the restraints according to their relative interaction score (interaction score divided by mean interaction score of all predicted contacts used).

Restrains from secondary structures elements were implemented as dihedral angle restraints. Dihedral angles of both beta strands and alpha helices were set to range between values commonly observed in crystal structures ${ }^{72}$, for alpha helices $\Phi_{\alpha}=-63.5^{\circ} \pm 4.5^{\circ}$ and $\Psi_{\alpha}=$ $-41.5^{\circ} \pm 5^{\circ}$ and for beta strands $\Phi_{\beta}=-118^{\circ} \pm 10.7^{\circ}$ and $\Psi_{\beta}=134^{\circ} \pm 8.6^{\circ}$.

1292 Restraints for beta sheet interactions were implemented by setting $\mathrm{H}-\mathrm{N}: \mathrm{O}=\mathrm{C}$ hydrogen bond 1293 distances between interacting positions to range between 1.8 and $2.1 \AA$, with weight one. 1294 Predictions of beta sheet interactions derived from deep mutational scanning data yield a string 1295 of interacting positions, but hydrogen bonding in beta sheets occurs in specific non-continuous 1296 patterns between position pairs (between alternating positions off the interaction diagonal in 1297 parallel beta sheets and between every second set of position pairs in anti-parallel beta sheets). 1298 Specifically, for each set of interacting positions there are two alternative patterns of hydrogen 1299 bonding possible. These alternative possibilities of pairing were implemented as mutually 1300 exclusive selection pairs with the "assign ... or" syntax in Xplor-NIH. 
Distance restraints were implemented in XPLOR-NIH as NOE (nuclear Overhausser effect) potential, with potential type set to "soft" for stages 1 and 2 and "hard" for the final simulation stage. Dihedral angle restraints were implemented via the CDIH potential.

1304 After simulation stages 1 and 2 restraints were checked for their consistency with predicted 1305 structural models. First, structural models were clustered according to their violations of 1306 distance and dihedral angle restraints ( $k$-means clustering, $k=4$ ). Clusters were ranked by the 1307 mean total energy (from all energy potentials used) of their 50 models with lowest total energy 1308 (or all of their structures if clusters are smaller 50 models). From the 50 models with the lowest 1309 total energy from the top-ranked cluster (or however many top-ranked clusters were necessary to arrive at 50 models) the fraction of models that violate specific restraints was recorded. For the subsequent simulation stage, distance restraints were down-weighted according to the fraction of models that violated them, $w_{x, i}=w_{x, i-1} *\left(1-f_{x}\right)^{2}$, and distance restraints with a weight below 0.1 were discarded. There is no option to weight dihedral angle restraints, thus instead dihedral angle restraints with a 'weight' below 1/3 were discarded for the subsequent simulation stages.

1316 The top 5\% structural models from simulation stage 3 were evaluated against the reference 1317 structure. The TM-score program (update 2016/03/23) was used to calculate the Ca root mean 1318 squared deviation and the template modeling score ${ }^{73}$.

1319 Several types of control simulations were performed to judge the predictive power of restraints 1320 derived from deep mutational scanning data. As a negative control we performed simulations 1321 without restraints from predicted contacts and beta sheet interactions, but with restraints from 1322 secondary structure elements predicted by PSIPRED (version $3.3,{ }^{43}$ ). As a positive control we 1323 performed simulations with restraints derived from the reference structure. Here, $L$ true contacts 1324 of position pairs with linear chain distance greater than 5 amino acids were randomly sampled 1325 and beta sheet interactions were determined by PyMOL ${ }^{44}$. These simulations serve as a 1326 positive control and give the maximally achievable accuracy of our Xplor-NIH workflow.

1327 For the WW domain, simulations on the full mutated 33aa section gave mediocre results, both

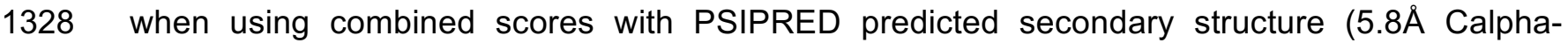
1329 RMSD), as well as when using perfect information from the reference structure (4.1A Calpha1330 RMSD). Upon inspection, this seemed to be an issue of the unstructured tail regions. We thus 1331 conducted structural simulations for a truncated version of the WW domain using only mutated 1332 positions 6-29 (the core region including the three beta strands). 
1333 For structural simulations of down-sampled GB1 datasets (and DeepContact transformed versions thereof) we used distance restraints derived from top predicted contacts and secondary structure restraints derived from PSIPRED predictions, but no restraints for beta sheet pairing. This was done to avoid skewed results due to false beta sheet pairing predictions in low quality datasets (Extended Data Figure 7B). For structural simulations from DeepContacttransformed predictions, we find that using more tertiary contacts results in better models. We conclude that this is because the deep learning algorithm focuses many strong predictions in few structural features (such as interactions of secondary structure elements), which are therefore the top contacts. Restraints in other regions of the protein are therefore only included if more predicted contacts are used for restraint calculations, therefore improving structural predictions. Because of this, when comparing structural simulations from scores derived before and after deep learning, we compare the top $5 \%$ of structural models derived with the top $L$ predicted contacts from original scores with those derived with the top $1.5^{*} \mathrm{~L}$ predicted contacts from DeepContact transformed scores.

\section{DeepContact learning}

1349 DeepContact software was obtained from GitHub (https://github.com/largelymfs/deepcontact) ${ }^{47}$. 1350 We are grateful to Yang Liu and Jian Peng for also making - without any hesitation - their basic 1351 DeepContact network architecture available on their GitHub repository and helping us with the 1352 implementation. The DeepContact architecture used here only takes one 2D input of predicted 1353 contact scores and returns a 2D map of transformed scores (denoted as "DeepContact 1354 CCMPred only" in ${ }^{47}$ and described in the first paragraph of the result section therein). The 1355 DeepContact architecture employed came with a pre-trained network model that had been 1356 trained on solved structures of the 40\% homology filtered ASTRAL SCOPe 2.06 database (see 1357 GitHub repository and Liu, et al. ${ }^{47}$ ), which were filtered to avoid structure and sequence 1358 redundancy of the training data. Because CCMpred scores ${ }^{74}$ are distributed in the range of 0 to 1359 1, we pre-normalized our deep mutational scanning derived interaction scores to this range 1360 (such that the minimum score on the interaction score matrix was 0 and the maximum score was 1) before providing them as an input to DeepContact. As negative control, we created for each dataset three random permutations of combined score matrices (while preserving matrix symmetry; in case of FOS-JUN dataset non-symmetric epistasis score matrices were permutated), which were transformed by the DeepContact algorithm. These control datasets show no increased precision of random expectation (Figure 6F). 


\section{Code availability}

1368 Data was analyzed with custom scripts written and executed in $\mathrm{R}$ programming language, 1369 version 3.4.3. Structural simulations were performed with Xplor-NIH modeling suite version 1370 2.46. Analysis scripts are available at https://github.com/lehner-lab/DMS2structure.

\section{Data availability}

1373 No primary data was generated in this study. Processed interaction scores for all datasets are 1374 included in Supplementary Table 1. All intermediate steps of data processing can be 1375 recapitulated with the scripts at https://github.com/lehner-lab/DMS2structure. 
bioRxiv preprint doi: https://doi.org/10.1101/303875; this version posted Auqust 18, 2018. The copyright holder for this preprint (which was not certified by peer review) is the author/funder, who has granted bioRxiv a license to display the preprint in perpetuity. It is made available under aCC-BY-NC-ND 4.0 International license.

\section{Extended Data Figures}

\section{Extended Data Figure 1}

deep mutational scanning experiment

1) mutate protein of interest

2) competitive selection assay

3) genotype frequencies from sequencing

computational analysis

4) calculate fitness $F_{i}$ and error $\sigma_{F_{j}}$ from sequencing read counts

5) $10^{4} \times$ re-sampling procedure

5a) sample variant fitness from $f_{i} \sim N\left(F_{i}, \sigma_{i}\right)$ \& classify epistasis (see panel $C$ )

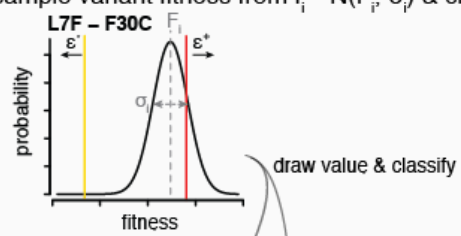

5b) aggregate per position pair \& sample epistatic fractions
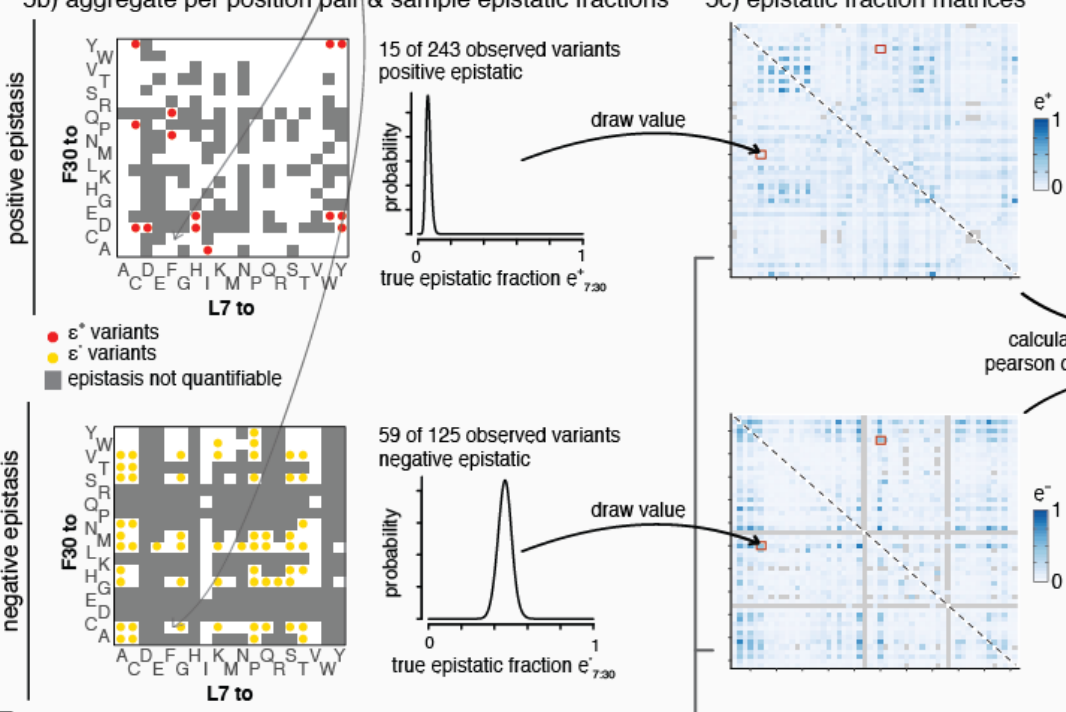

5d) correlation of epistasis patterns

6) compute interaction scores by

merging positive \& negative epistasis information
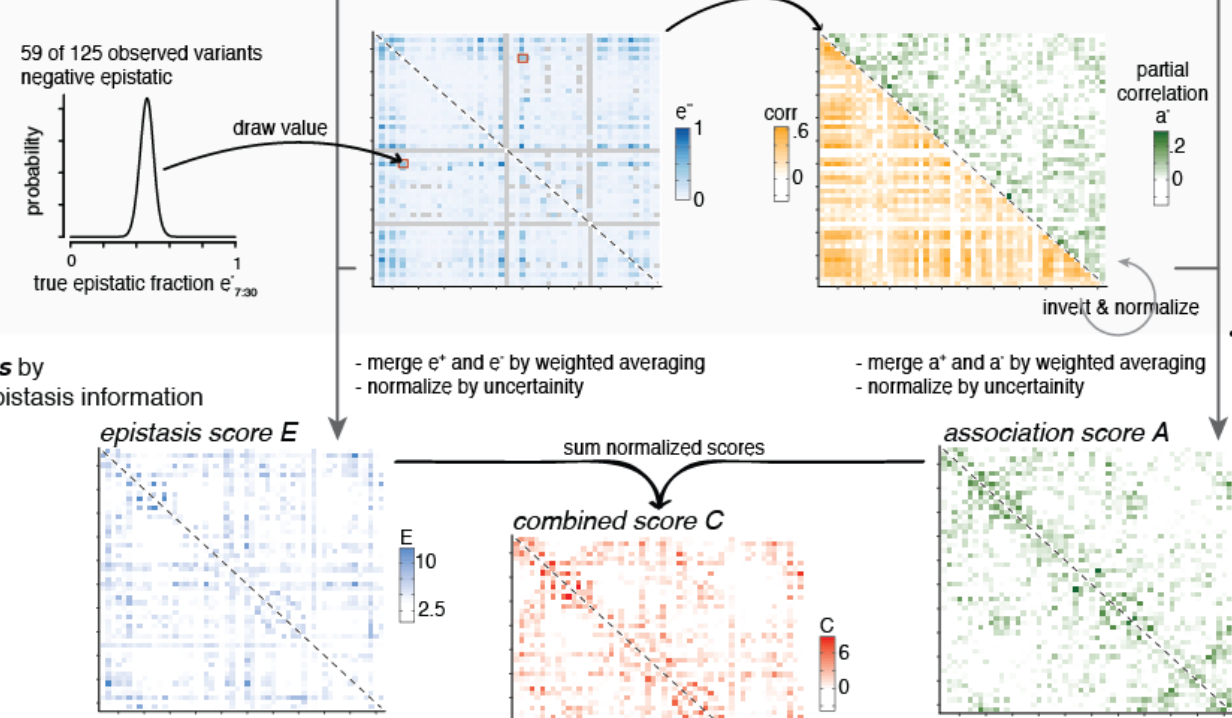

merge $\mathrm{a}^{+}$and $\mathrm{a}^{*}$ by weighted averaging normalize by uncertainity

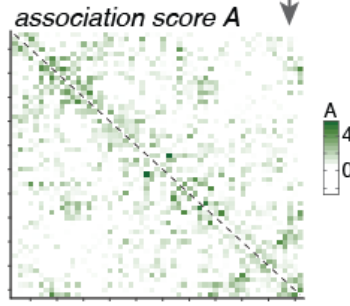


bioRxiv preprint doi: https://doi.org/10.1101/303875: this version posted Auqust 18. 2018. The copvriaht holder for this preprint (which was not

certified by peer review) is the author/funder, who has granted bioRxiv a license to display the preprint in perpetuity. It is made available under aCC-BY-NC-ND 4.0 International license.

1378 Extended Data Figure 1: Deep mutational sequencing data to contact 1379 prediction workflow

1380 Overview of workflow to predict interacting position pairs from deep mutational scanning

1381 datasets (see Methods and Results).

1382 
bioRxiv preprint doi: $\mathrm{https}$ //doi.org/10.1101/303875; this version posted August 18,2018 . The copyright holder for this preprint (which was not certified by peer review) is the author/funder, who has granted bioRxiv a license to display the preprint in perpetuity. It is made available under aCC-BY-NC-ND 4.0 International license.

\section{Extended Data Figure 2}

\section{A}

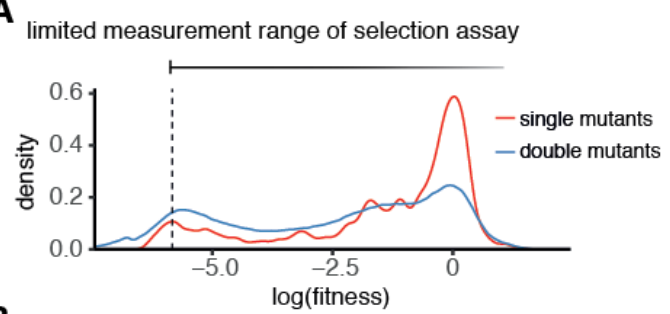

B discarding variants with low input coverage

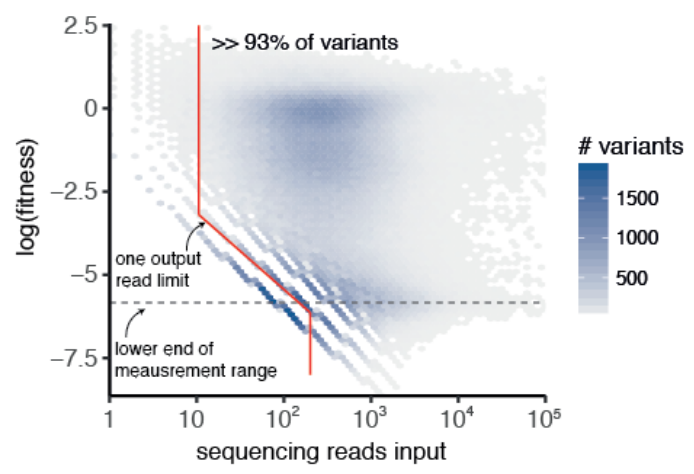

C data subsets suitable for epistasis classification

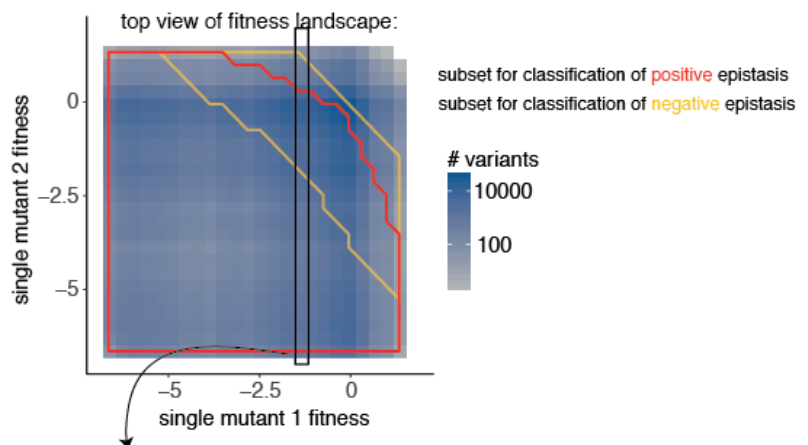

1383

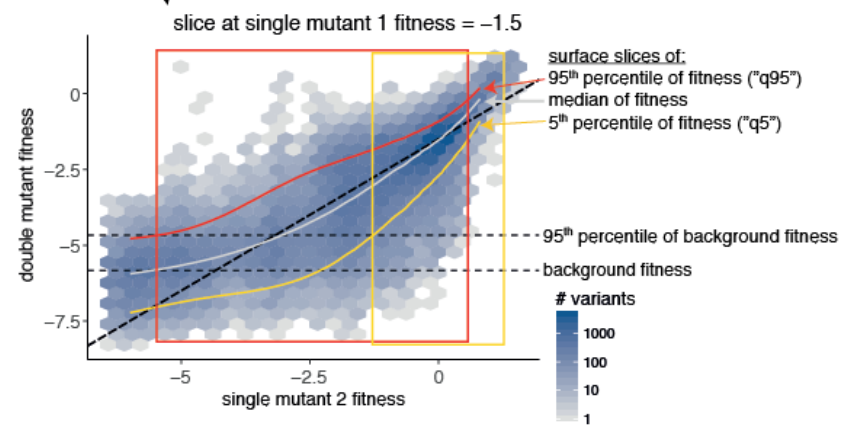

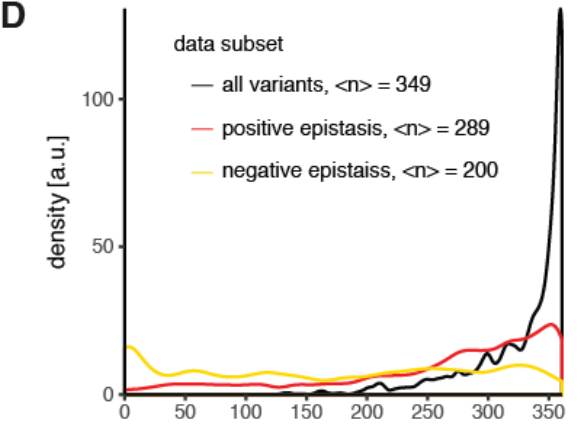

number of double mutants per position pair

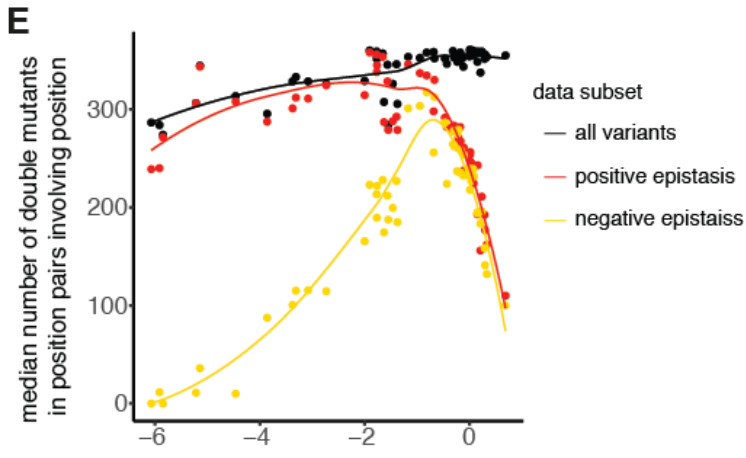

median single mutant fitness at position

$\mathbf{F}$ negative epistasis quantifiaction

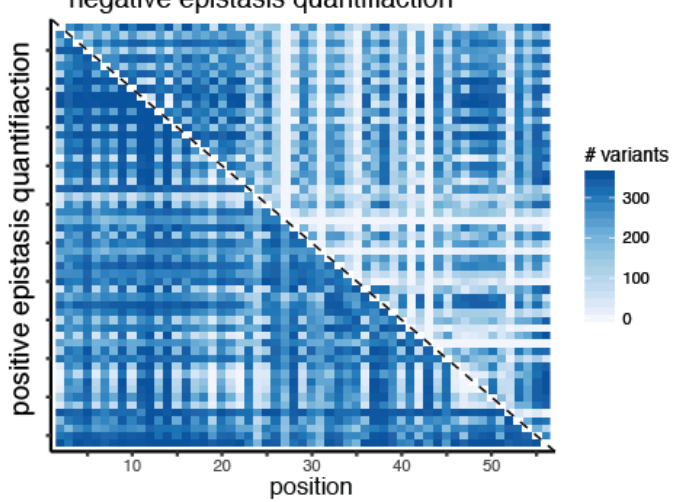

1384 Extended Data Figure 2: GB1 deep mutational scanning data processing

A. Distribution of fitness values for single and double mutant variants. Lower peak in distributions indicates lower limit of fitness assay measurement range (see Methods). 
B. Two-dimensional variant density showing dependency of fitness values on sequencing read counts in input library. For variants with very low coverage in the input library low fitness values cannot be accurately estimated. Red line shows sequence read cutoff used for variant inclusion (93\% of variants included for downstream analysis). Horizontal dashed line indicates lower limit of fitness assay measurement range.

C. Reliable quantification of positive and negative epistasis is limited to subsets of the data. Upper plot shows two-dimensional double mutant variant density in single mutant fitness space. Red outline shows single mutant fitness space that enables positive epistasis quantification. Yellow outline shows single mutant fitness space that enables negative epistasis quantification. Lower plot shows an example slice through fitness landscape at single mutant fitness $=-1.5$. Red, grey and yellow curves show slices through quantile fitness surfaces of $95^{\text {th }}$ percentile, median and $5^{\text {th }}$ percentile, respectively (see Figure 1B).

Diagonal dashed line shows double mutant fitness $=$ single mutant 2 fitness - 1.5 (expected fitness = observed fitness). Horizontal dashed lines give lower limit of fitness assay measurement range and the $95^{\text {th }}$ percentile of fitness values of variants dominated by background fitness effects. Red and yellow boxes indicate the range that includes $99 \%$ of variants within the slice that are suitable for positive or negative epistasis quantification, respectively.

D. Distribution of number of double mutant variants across all position pair. Legend gives median number of double mutants per position pair for different data subsets. of double mutants observed in position pairs the position is involved in. Curves are loess smoothed. Across all variants, positions with stronger fitness effects show lower coverage of double mutants. Restrictions for quantification of positive epistasis additionally reduce coverage for positions with mostly neutral or positive effects. Finally, restrictions for quantification of negative epistasis strongly reduce coverage for positions with strong fitness effects, due to the lower measurement limit of the fitness assay. 


\section{Extended Data Figure 3}

A

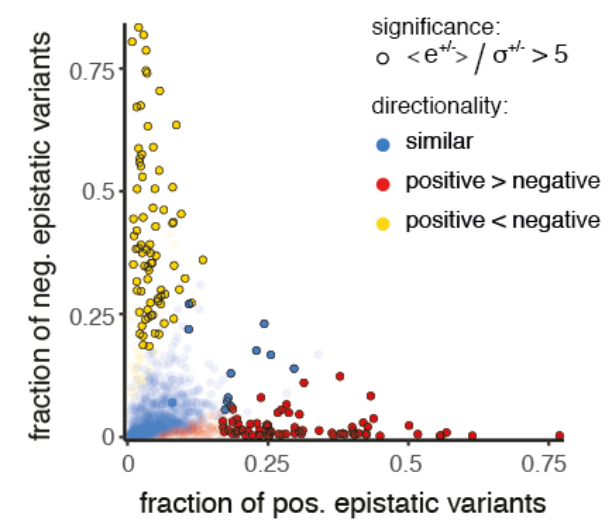

B

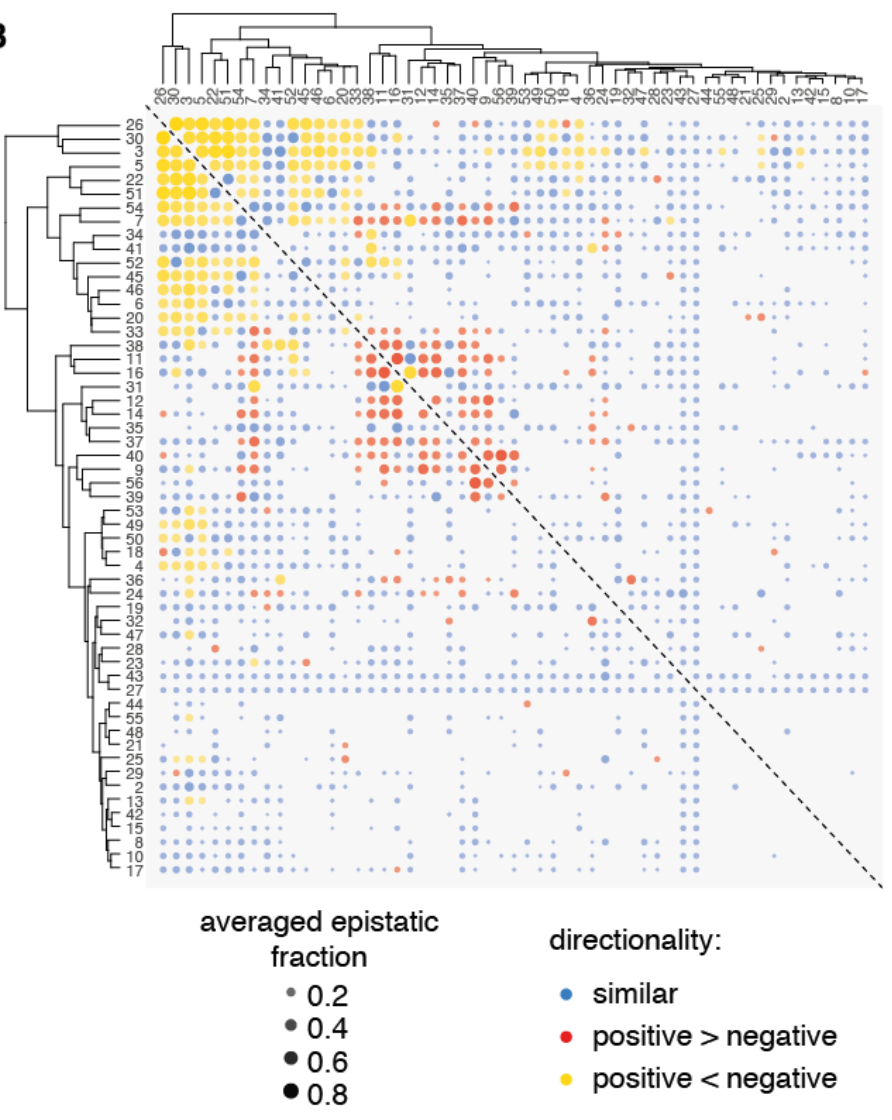

1418 Extended Data Figure 3: Positive and negative epistasis enrichments across position pairs

A. Position pair-wise fractions of positive and negative epistatic variants. Black circles mark position pairs with highly significant fractions (either positive or negative, or both); red dots: positive epistatic fraction significantly larger than negative epistatic fraction; yellow dots: negative epistatic fraction significantly larger than positive epistatic fraction; blue dots: no significant differences.

B. Hierarchical cluster analysis of epistatic fraction patterns. Positions are clustered according to the Euclidean distance of their mean epistatic fractions (weighted average of positive and negative epistatic fractions, weights are inverse variances of fractions in resampling runs) to all other positions. Note that directionality of interactions (positive or negative fractions more significant) was not used for clustering but only marked post-analysis. Clustering shows two highly interconnected clusters of positions that interact mostly positively or 
bioRxiv preprint doi: https://doi.org/10.1101/303875; this version posted August 18,2018 . The copyright holder for this preprint (which was not certified by peer review) is the author/funder, who has granted bioRxiv a license to display the preprint in perpetuity. It is made available under aCC-BY-NC-ND 4.0 International license. 
bioRxiv preprint doi: https://doi.org/10.1101/303875; this version posted August 18, 2018. The copyright holder for this preprint (which was not certified by peer review) is the author/funder, who has granted bioRxiv a license to display the preprint in perpetuity. It is made available under aCC-BY-NC-ND 4.0 International license.

\section{Extended Data Figure 4}

A

detecting parallel \& anti-parallel $\beta$-sheets

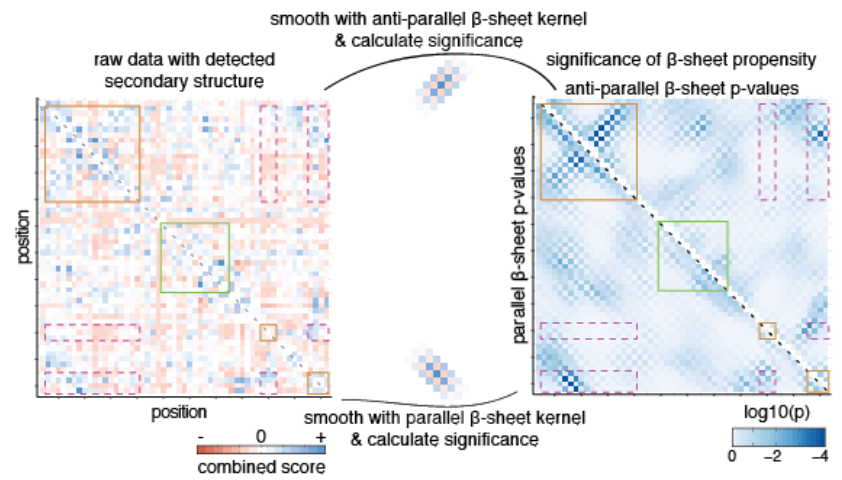

B

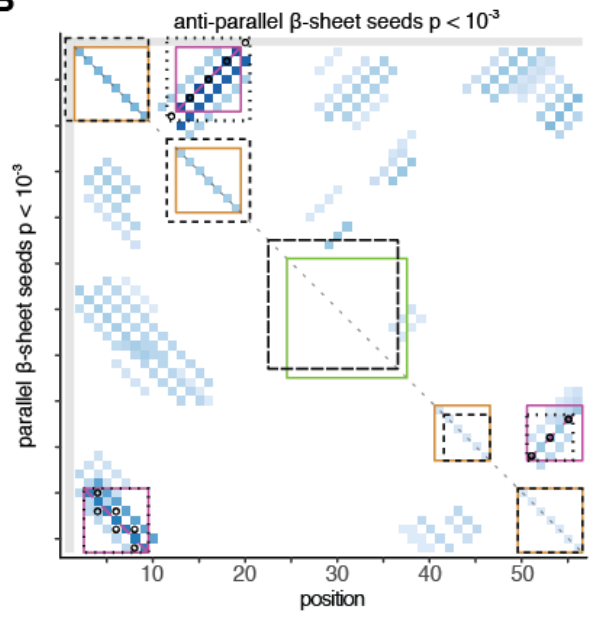

$\mathbf{F}$

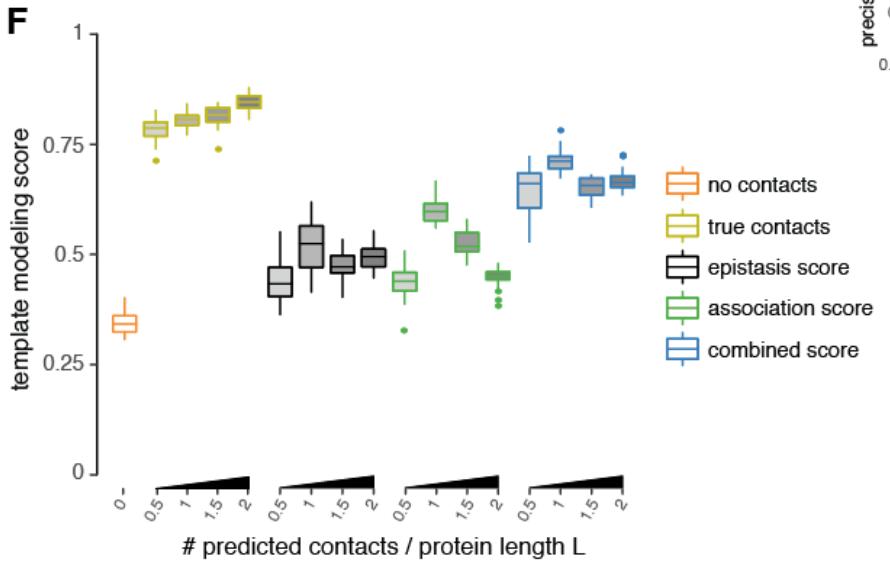

1435 $\beta$-strand
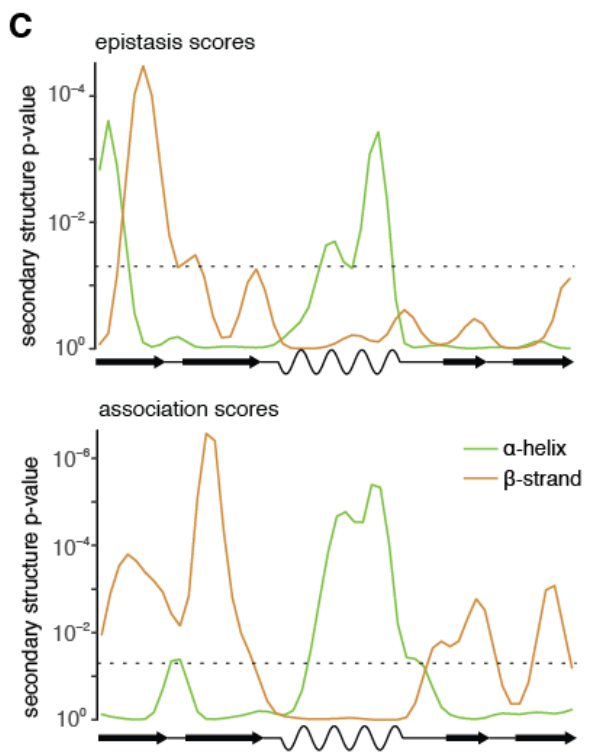

D - a-alpha helix . $\beta$-sheet interaction

$\log 10(p)$

$0-5-10$ al predictions

a-helix

$\beta$-strand

$\beta$-sheet interaction
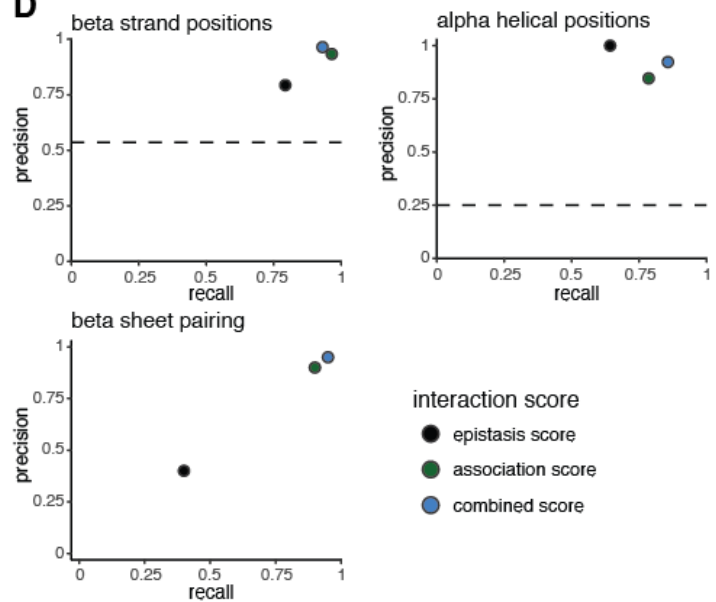

interaction score

- epistasis score

association score

combined score
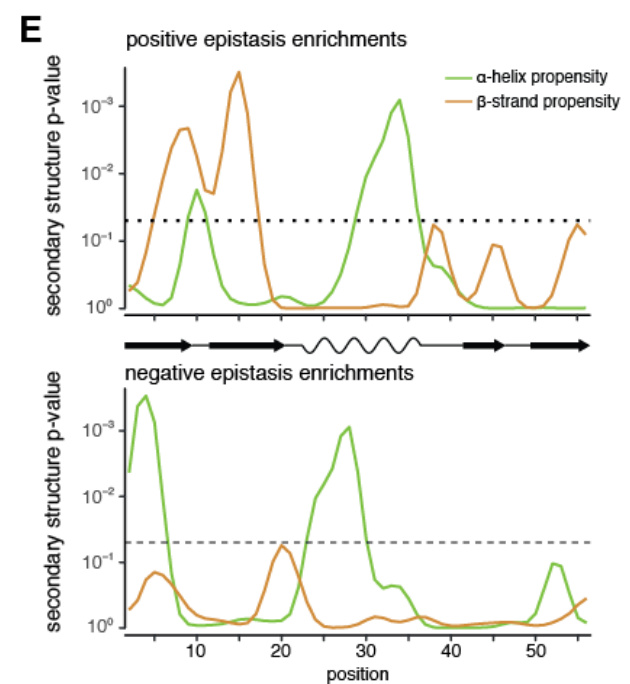


\section{Extended Data Figure 4: Secondary and tertiary structure prediction for}

1437 GB1 domain

1438 A. Detecting beta sheet pairing with two-dimensional kernel smoothing. Left plot shows raw combined score interaction matrix, with secondary structure element predictions (see Figure 4A,B) marked as squares along the diagonal (red - beta strand, green - alpha helix). Off-diagonal orange rectangles show potential regions of beta sheet pairing. Right plot: calculation of beta sheet pairing propensity with beta sheet kernels. Upper right triangle shows anti-parallel beta sheet propensity. Lower left shows parallel beta sheet propensity.

B. Matrix of aggregated propensities of beta sheet pairing stretches (upper right - antiparallel, lower left - parallel, $\mathrm{p}<10^{-3}$ ) and the predictions for beta sheet pairing and secondary structure elements derived from them. In brief, predictions are performed by picking the highest propensity stretch that is consistent with predicted beta strands, if necessary modifying beta strand predictions (e.g. introducing an initially not predicted split between beta strands 1 and 2), then disregarding all stretches that conflict with the picked top-stretch. This procedure is repeated until no more beta sheet stretches with propensity $\mathrm{P}<10^{-3}$ are left. Finally, beta strand predictions are updated such that only positions involved in a beta sheet interaction are retained. Reference elements from crystal structure are shown as comparison (lower triangle - parallel beta sheets, upper triangle - anti-parallel beta sheet, diagonal -secondary structure elements).

C. Secondary structure propensity derived from kernel smoothing (red - beta strand, green - alpha helix) for epistasis (upper) and association scores (lower). P-values were derived by comparison to randomized datasets (see Methods). Dashed line indicates $p=$ 0.05 .

D. Precision and recall for beta strand, alpha helix and beta sheet predictions from epistasis, association and combined scores (in comparison to crystal structure). Dashed lines for beta strand and alpha helical positions give random expectation. Random expectation for beta sheet pairing precision is below $1 \%$.

E. Secondary structure propensities derived from local positive or negative epistatic enrichments. The upper panel shows secondary structure propensity derived from positive epistatic interactions, which are in line with secondary structure elements in the GB1 crystal structure (PDB entry 1pga). The lower panel shows secondary structure propensity derived from negative epistatic interactions, which are devoid of beta strand 
signals and instead show a three-helical pattern, which is reminiscent of the three-helical structure of the protein $\mathrm{G} A$ domain that binds albumin ${ }^{57}$.

1471 F. Template modeling score of top $5 \%$ structural models compared to crystal structure $1 \mathrm{pga}$ and the dependency on number of predicted contacts used. "No contacts" - only restraints for secondary structure predicted by PSIPRED. "True contacts" - restraints derived from $0.5-2^{*} \mathrm{~L}$ contacts (linear sequence separation greater than 5 positions, random subset), secondary structure elements and beta sheet interactions from crystal structure. All other: restraints derived from top $0.5-2^{*} \mathrm{~L}$ contacts, secondary structure element and beta sheet interaction predictions from the three interaction scores, as indicated by color. 
bioRxiv preprint doi: https://doi.org/10.1101/303875; this version posted August 18,2018 . The copyright holder for this preprint (which was not certified by peer review) is the author/funder, who has granted bioRxiv a license to display the preprint in perpetuity. It is made available under aCC-BY-NC-ND 4.0 International license.

\section{Extended Data Figure 5}

A ww domain

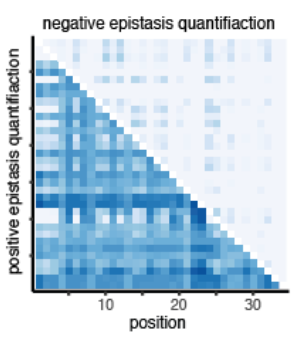

B

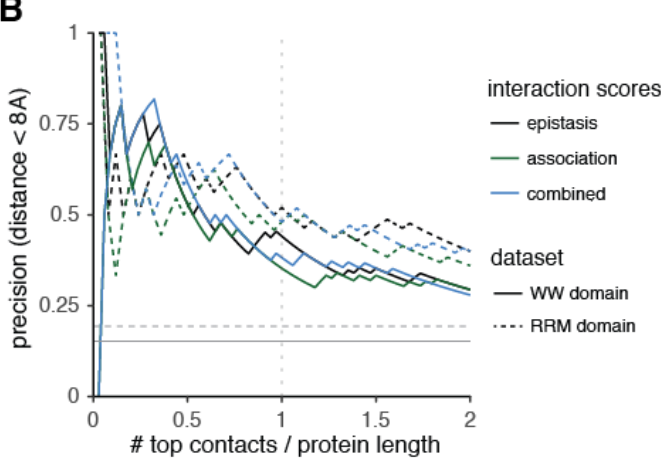

C

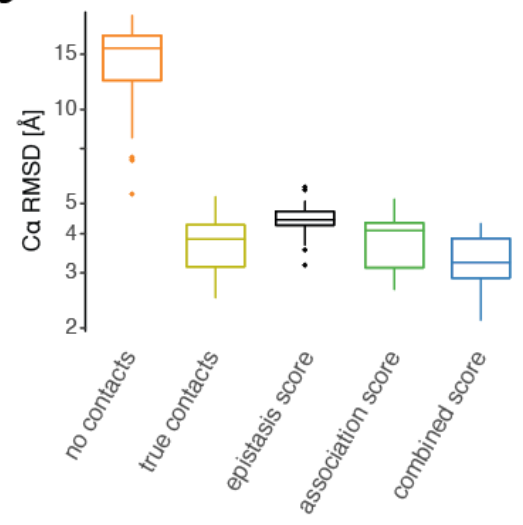

RRM domain

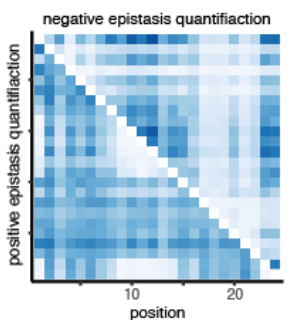

sition

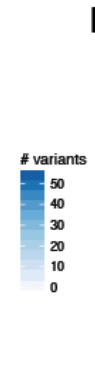

D FOS-JUN interaction
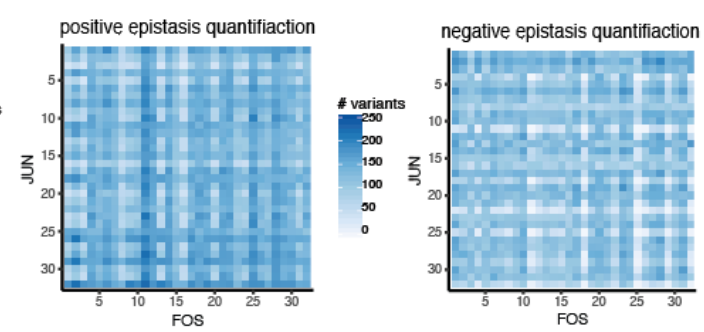

E before anter

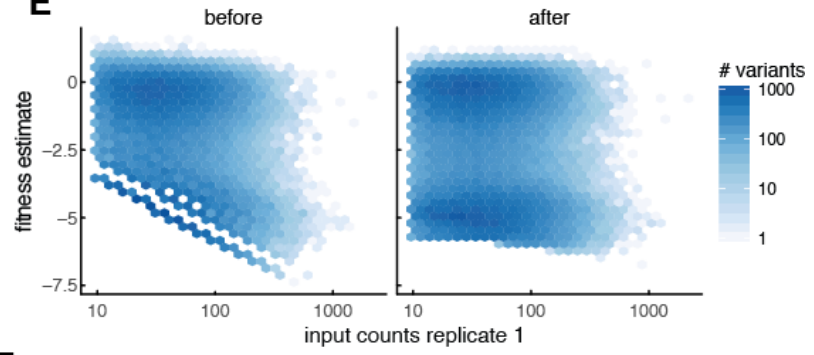

$\mathbf{F}$

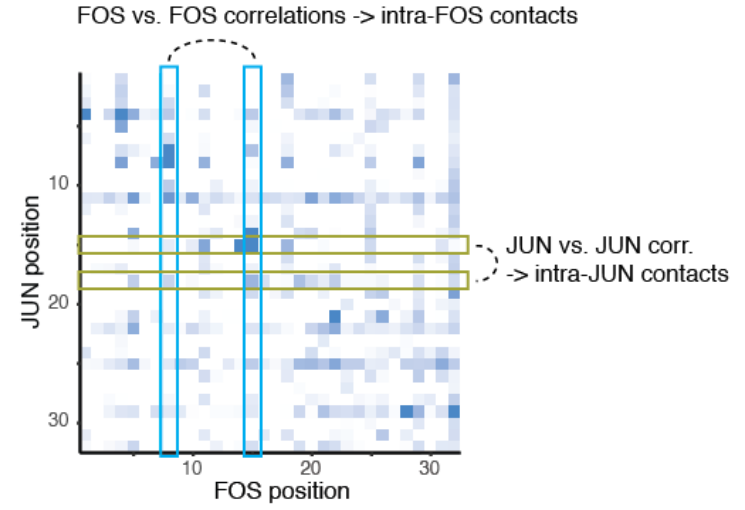

G

intra-FOS

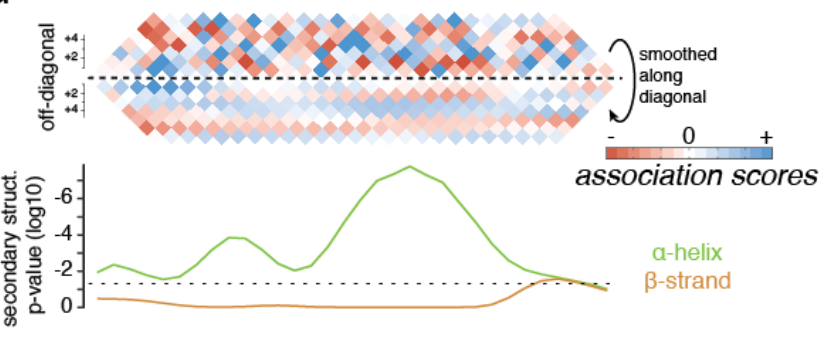

intra-JUN
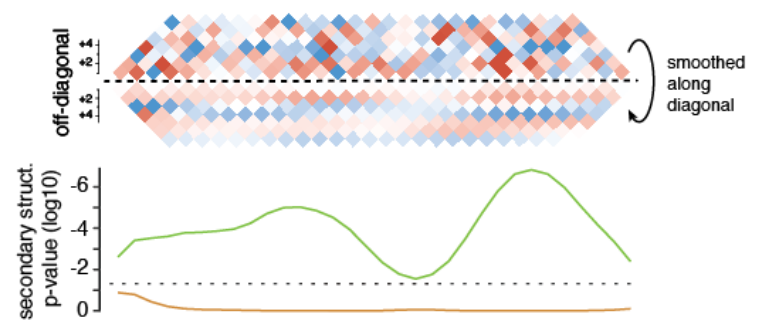


\section{Extended Data Figure 5: WW and RRM domain and FOS-JUN}

1482 interaction dataset properties

A. Number of double mutants for which positive (lower left triangle) or negative (upper right triangle) epistasis can be quantified per position pair plotted on the interaction matrix for WW (left) and RRM (right) domains.

B. Precision of interaction scores to predict direct contacts (distance $<8 \AA$ in reference structure) as a function of top scoring position pairs for WW and RRM domain interaction scores. Color denotes interaction scores, solid lines for WW domain, dashed lines for RRM domain. Grey horizontal lines give random expectation. Only position pairs with linear sequence separation greater than 5 amino acids are considered.

C. Accuracy ( $C \alpha$ root-mean-square deviation) of top $5 \%$ structural models of the WW domain (core positions 6-29) generated from deep mutational scanning data derived restraints compared to reference structure (PDB entry $1 \mathrm{k} 9 \mathrm{q}$ ). Structural models were generated in XPLOR-NIH by simulated annealing with restraints derived from top 17 tertiary contacts and secondary structure elements predicted by PSIPRED. No beta sheet pairing information was used.

D. Number of double mutants for which positive (left) or negative (right) epistasis can be quantified per position pair plotted on the trans-interaction matrix of the FOS-JUN interaction.

E. Bayesian estimation of fitness values in FOS-JUN interaction data. Mutants with low input sequencing coverage display limited measurement range and many dropouts ( $\sim 15 \%$ of variants without reads in output). Left panel shows original fitness distribution as function of input coverage in replicate 1, right panel shows Bayesian estimates of fitness as function of input coverage in replicate 1.

F. Learning about intra-molecular contacts in FOS or JUN from epistatic pattern correlations. Column-wise correlation of epistatic patterns of the trans interaction score map serve to calculate intra-FOS association scores and thus reveal relationships between positions in FOS. Likewise, row-wise correlation of epistatic patterns reveal relationships between positions in JUN. 
G. Local interactions in intra-molecular association scores reveal secondary structures of protein interaction partners. Upper panels: Data above diagonal shows association score data close to the diagonal, i.e. local interactions. Data below the diagonal is smoothed with a Gaussian kernel to reveal interaction periodicity. Lower panels: Secondary structure propensities derived from kernel smoothing (see Figure 4A-C). Green indicates alpha helical propensity, orange indicates beta sheet propensity, $p=0.05$ is indicated by dashed line. 


\section{Extended Data Figure 6}

A
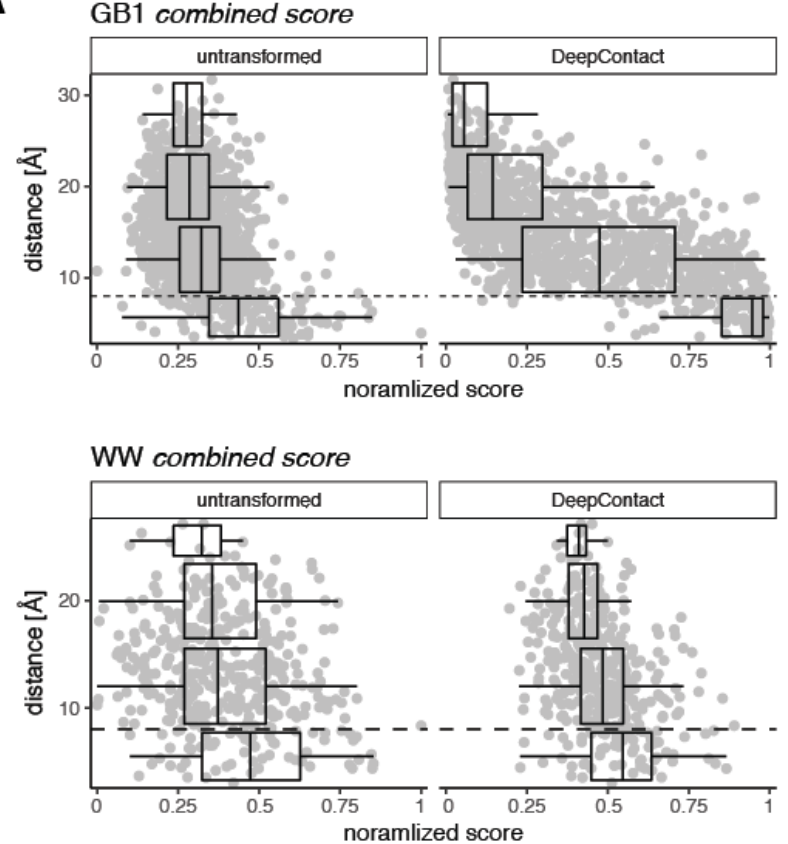
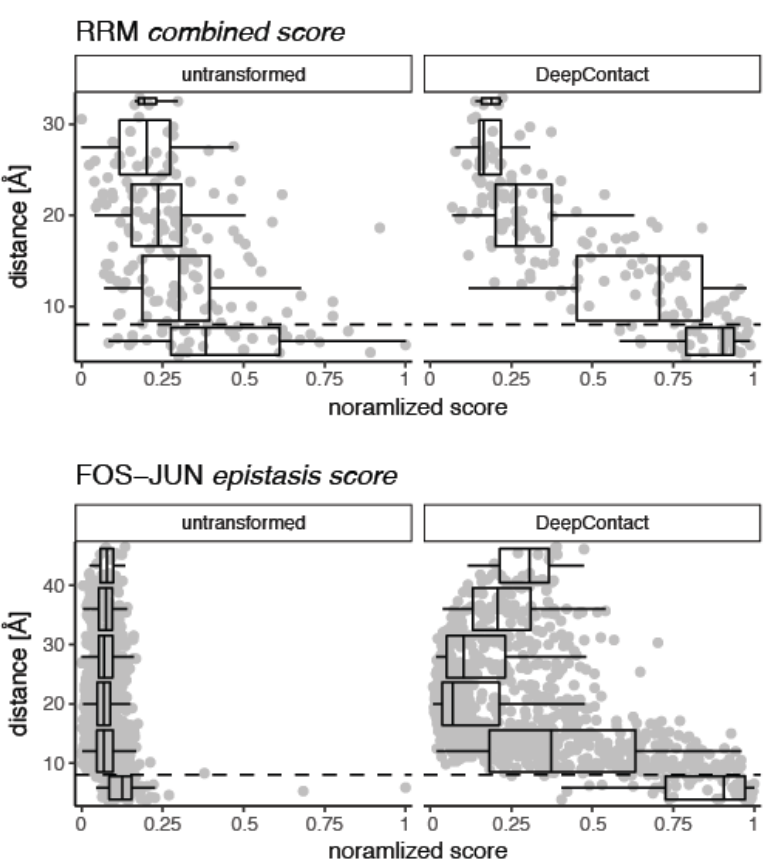

\section{B WW domain}

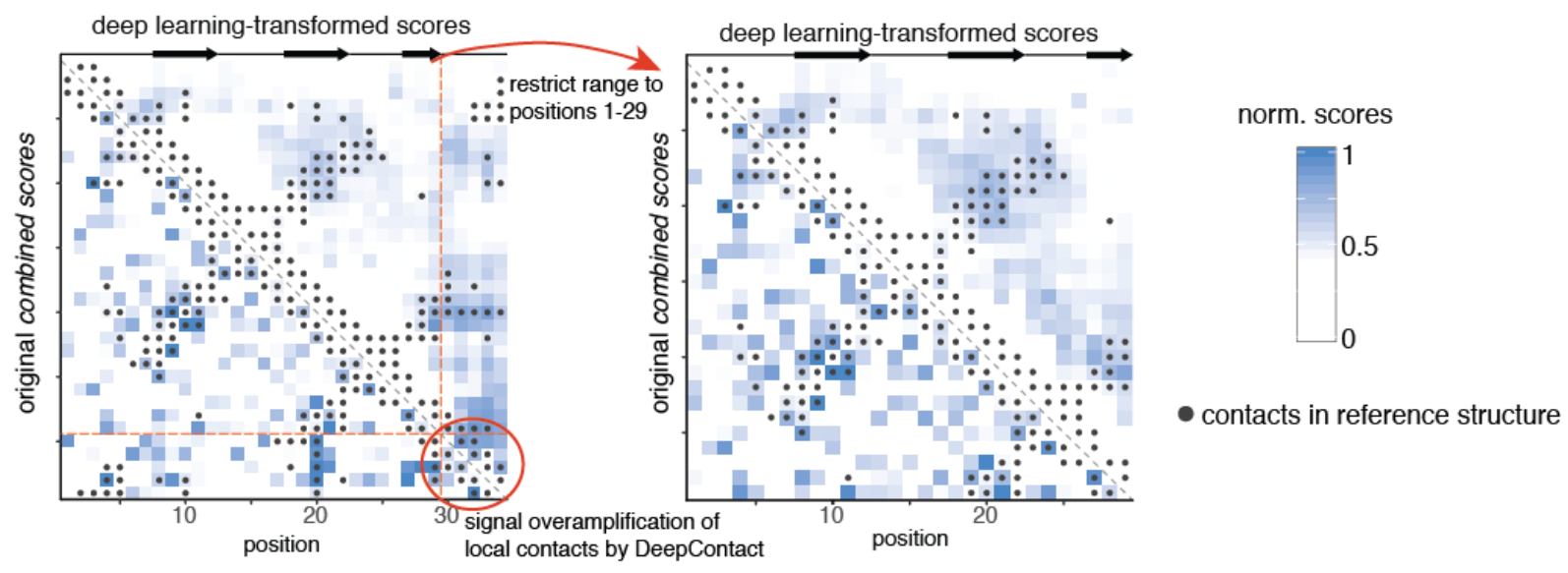

1519 Extended Data Figure 6: Deep learning improves contact

A. Distance of position pairs as a function of interactions scores before (left panel, scores normalized to interval $[0,1]$ ) and after (right panel) transformation with DeepContact for the four datasets. Boxplots are spaced in distance intervals $[0,8),[8,16),[16,24),[24,32)$, $[32,40)$ and $[40,48) \AA$. Dashed horizontal line indicates $8 \AA$.

B. Left: Full WW domain combined score interaction map before (lower left) and after (upper right) DeepContact transformation. DeepContact amplifies a signal from local 
bioRxiv preprint doi: https://doi.org/10.1101/303875; this version posted August 18,2018 . The copyright holder for this preprint (which was not certified by peer review) is the author/funder, who has granted bioRxiv a license to display the preprint in perpetuity. It is made available under aCC-BY-NC-ND 4.0 International license.

contacts in the C-terminal region of the domain, thus concentrating the strongest transformed signal in this region. Removing positions 30-34 removes this artefact (right plot). Heat maps show interaction scores that have been normalized to have similar range. Grey dots show contacts (distance $<8 \AA$ ) in reference structure. 
bioRxiv preprint doi: https://doi.org/10.1101/303875; this version posted Auqust 18 , 2018. The copyright holder for this preprint (which was not certified by peer review) is the author/funder, who has granted bioRxiv a license to display the preprint in perpetuity. It is made available under aCC-BY-NC-ND 4.0 International license.

\section{Extended Data Figure 7}

\section{A}

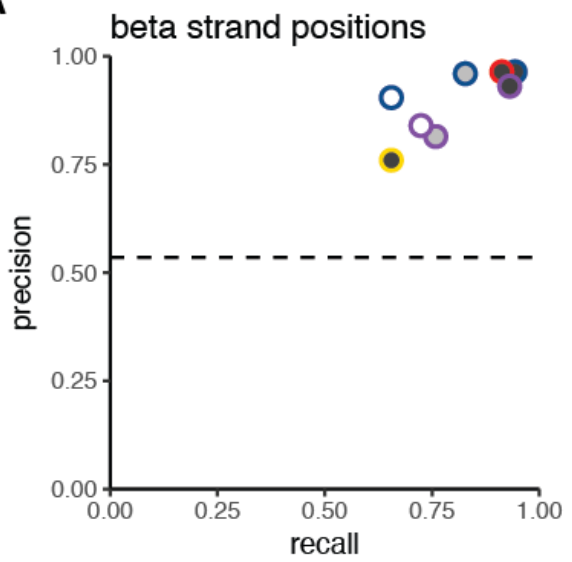

beta sheet pairing

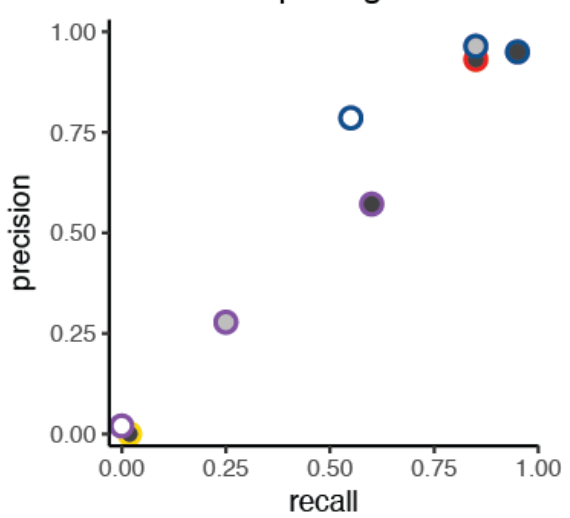

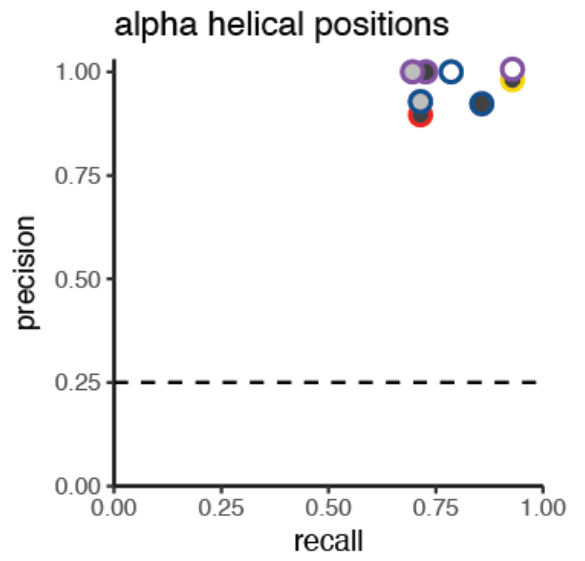

B GB1 doped dataset combined score

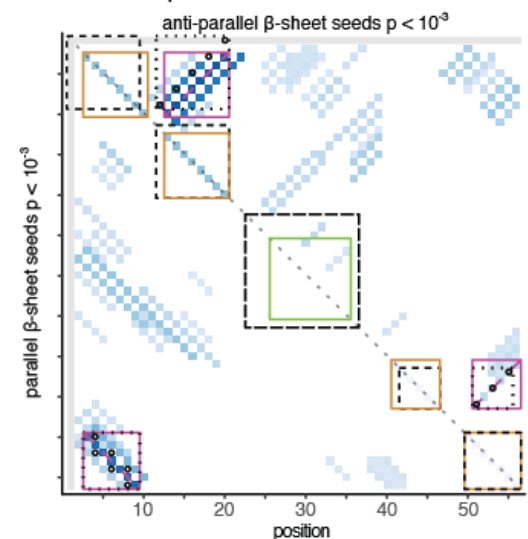

\#seq. reads

○ $100 \%$

○ $25 \%$

○ $10 \%$

dataset

O all variants

only 'doped' variants

only pos. epistatic variants

only neg. epistatic variants

final predictions

$\square$ a-helix

$\beta$-strand
$\beta$-sheet interaction

1pga crystal structure

[- $a$-alpha helix

-strand

$2 \beta$-sheet interaction

$\log 10(\mathrm{p})$

$-5-10$

C
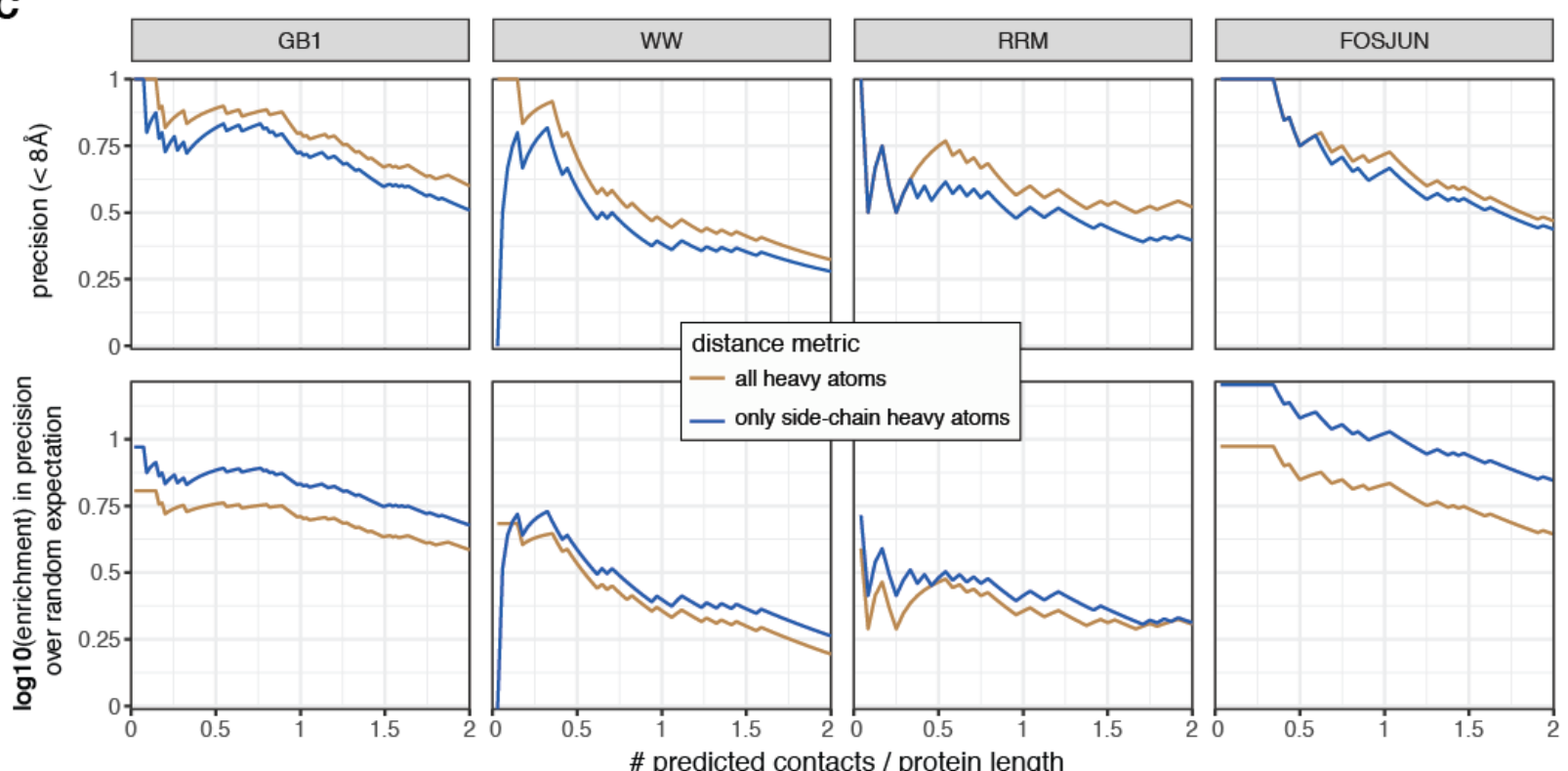

1532

\# predicted contacts / protein length

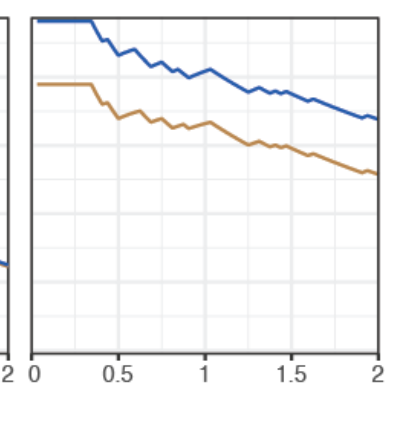




\section{Extended Data Figure 7: Distance metric comparison and}

1534 secondary structure prediction for lower data-quality GB1

1535 datasets

1536 A. Precision and recall for beta strand, alpha helix and beta sheet predictions derived from combined scores of down-sampled GB1 datasets (in comparison to reference structure). Dashed lines for beta strand and alpha helical positions give random expectation. Random expectation for beta sheet pairing precision is below $1 \%$. Note that some coinciding data points were slightly moved for better identifiability.

B. Beta sheet pairing predictions for the doped GB1 dataset with $100 \%$ sequencing read coverage (cf. Extended Data Figure 4B). Beta sheet pairing between beta strands 1 and 2 is predicted in correct anti-parallel direction, but exact pairing of positions are off by 2 ; thus precision and recall of beta sheet pairing for doped GB1 dataset drops to $\sim 60 \%$ (see panel B).

C. Differences in precision and enrichment over random expectation for all heavy atom or side-chain heavy atom distance metrics. As expected, using all heavy atoms (including backbone heavy atoms) increases precision of predicted contacts by about $10 \%$. Restricting distance measurements to side-chain heavy atoms, however, increases precision over random expectation, often by more than 2-fold (note the log10-scale), indicating that side-chain distances are more informative for epistatic interactions. For 